Pivovarov, D., Oberleiter, T., Willner, K. and Steinmann, P. (2019) Fuzzy-stochastic FEM-based homogenization framework for materials with polymorphic uncertainties in the microstructure. International Journal for Numerical Methods in Engineering, 116(9), pp. 633-660.

There may be differences between this version and the published version. You are advised to consult the publisher's version if you wish to cite from it.

This is the peer reviewed version of the following article:

Pivovarov, D., Oberleiter, T., Willner, K. and Steinmann, P. (2019) Fuzzy-stochastic FEM-based homogenization framework for materials with polymorphic uncertainties in the microstructure. International Journal for Numerical Methods in Engineering, 116(9), pp. 633660.(doi: 10.1002/nme.5947)

This article may be used for non-commercial purposes in accordance with Wiley Terms and Conditions for Self-Archiving.

http://eprints.gla.ac.uk/185714/

Deposited on: 13 June 2019

Enlighten - Research publications by members of the University of Glasgow http://eprints.gla.ac.uk/ 


\title{
Fuzzy-Stochastic FEM-based homogenization framework for materials with polymorphic uncertainties in the microstructure
}

\author{
Dmytro Pivovarov*, Thomas Oberleiter, Kai Willner, and Paul Steinmann \\ Chair of Applied Mechanics, University of Erlangen-Nuremberg, 91058 Erlangen, Germany
}

Correspondence: *Dmytro Pivovarov, Chair of Applied Mechanics, University of Erlangen-Nuremberg, Paul-Gordan-Str. 3, 91052 Erlangen, Germany. Email: dmytro.pivovarov@fau.de

Received ; Revised ; Accepted

\begin{abstract}
Summary
Uncertainties in the macroscopic response of heterogeneous materials result from two sources: the natural variability in the microstructure's geometry and the lack of sufficient knowledge regarding the microstructure. The first type of uncertainty is denoted aleatoric uncertainty and may be characterized by a known probability density function. The second type of uncertainty is denoted epistemic uncertainty. This kind of uncertainty cannot be described using probabilistic methods. Models considering both sources of uncertainties are called polymorphic. In the case of polymorphic uncertainties some combination of stochastic methods and fuzzy arithmetic should be used. Thus in the current work we examine a fuzzy-stochastic FEM-based homogenization framework for materials with random inclusion sizes. We analyze an experimental radii distribution of inclusions and develop a stochastic representative volume element (RVE). SFEM is used to obtain the material response in the case of random inclusion radii. Due to unavoidable noise in experimental data, insufficient number of samples and limited accuracy of the fitting procedure the radii distribution density cannot be obtained exactly, thus it is described in terms of fuzzy location and scale parameters. The influence of fuzzy input on the homogenized stress measures is analyzed.
\end{abstract}

Keywords: Stochastic FEM, Stochastic local basis, Fuzzy numbers, Computational Homogenization, Geometrical uncertainties

\section{Introduction}

The effective macroscopic properties of heterogeneous materials are estimated from the response of the underlying microstructure by homogenization. Homogenization techniques proposed in the literature can be classified based on the considered physical model (deterministic or uncertain) or based on the applied homogenization method (analytical or computational). 
Some examples of analytical homogenization of deterministic media can be found in (3, 4). Interesting results in computational homogenization of deterministic media are presented in $(14,34,36,47,54,78)$. For an overview of existing deterministic homogenization techniques we refer to (70).

Real heterogeneous materials always possess either uncertain material properties, or some kind of geometrical uncertainties in the microstructure, or both types. In many cases the influence of these uncertainties cannot be neglected without some loss of accuracy.

The uncertainties in the microstructure result from two different sources. Aleatoric uncertainty originates from the natural variability of the microstructure. It may be described using the concept of probability. The required probabilistic measures of the model's input like, e.g., probability density functions, correlation functions, and probabilistic parameters can be estimated statistically from experimental data. Epistemic uncertainty derives from insufficient knowledge regarding the microstructure and parameter distribution, imperfection of the analytical models, and the experimental limitations. In this case we cannot assign probability measures to the model's input data $(\underline{8,76})$, instead the possibilistic approach is involved (89). This is an alternative to the probability operating not with random variables but with fuzzy numbers. Analogously to the probability density function the possibility density function is introduced (89), which is equivalent to the membership function. Axiomatic formulation of the possibility theory and its comparison with probability theory are presented in (56). The possibilistic approach is based on the concept of fuzzy sets introduced for the first time in (90). Later it was modified and applied to engineering problems involving epistemic uncertainty $(27,-29,31,32,41,42)$. For an overview of existing techniques we refer to (53). If the uncertainties result from both sources, the term polymorphic uncertainty is used.

As already mentioned, it is well appreciated that there are two different types of uncertainties possessing different nature. The aim of this paper is not to compare probabilistic and possibilistic approaches but to demonstrate by an example of real materials these two sources of uncertainties and to include them into the material model suitable for computational homogenization. Thus the focus of the paper is the development of a mathematical model and fitting this model to the experimental data. We use different modeling techniques for different types of uncertainties, thus we believe that both approaches, stochastic analysis and fuzzy analysis, are in general not concurrent but complementary (section 3.1).

In many studies dedicated to homogenization of heterogeneous materials with uncertainties in the microstructure only aleatoric uncertainty is considered, thus these techniques are called stochastic homogenization methods.

A number of results in analytical stochastic homogenization were obtained in $(5,13,23,71)$. Here we would like to highlight also the rapidly developing general perturbation method (37). Nowadays the perturbation method is one of the most popular stochastic techniques. Very promising results are obtained by combining perturbation method with smoothed FEM (48), with multiscale FEM (12), etc. Recently the general perturbation method was also successfully applied to the problem with geometrical uncertainties (39) in a setting very close to the one studied in this paper. The idea of classical perturbation methods is 
close to the Taylor series expansion thus inheriting its convergence properties. However, nowadays the more general setting is presented.

Different aspects and applications of stochastic computational homogenization were reported in (2, 15, 17, 19, 22, 46, 49, 51, 74, 79, 80, 82, 86, 88).

The two most universal stochastic methods (within the fully computational approach) are the Monte-Carlo simulation (MC) and the stochastic FEM (SFEM). Under SFEM we here understand the stochastic Galerkin based technique initially proposed in (25). Further modifications of these techniques were reported, e.g., in (1, 7, 9, 11, 18, 26, 33, 44, 45, 52, 55, 57, 62, 68, 72, 85). The main attraction of SFEM is the fact that it solves the problem only once, providing immediately the solution for the whole continuum of different realizations of the stochastic process (25). SFEM was already successfully applied to problems involving diffusion, stochastic nanomechanics, stochastic plasticity, and thermo-mechanics $(21,43,66,67,83)$. For an overview of the existing applications and modifications we refer to $(\underline{81)}$ and $(\underline{6})$.

It is clear that uncertainties in the microstructure are in most cases polymorphic, thus some combination of the fuzzy approach and the stochastic approach should be established. In this work we carefully analyze the experimental data and scanning electron microscopy pictures of heterogeneous materials (section 4) and demonstrate the sources of aleatoric and epistemic uncertainties (section 4.1 and 4.2). A combined fuzzy-stochastic FEM approach is used to perform simulations of fuzzy-stochastic representative volume elements (section 3). Aleatoric uncertainty is treated using stochastic FEM. In the present study we choose the SFEM formulation involving local finite element approximations of the physical and stochastic domains, which was found to be most accurate for the problem considered (65). Fuzzy parameters are treated using the general transformation method for fuzzy numbers. Based on the obtained results (section 5 and 6) we estimate the influence of the epistemic uncertainty on the homogenized quantities and obtain the lower and the upper bounds of the stress curves. Finally, section 7 concludes the paper.

\section{Notation}

In this work we distinguish between deterministic and random variables, vectors and tensors, matrices and operators. We use the following notation:

- Second order tensors and vectors are denoted by bold (e.g. F) and bold italic (e.g. $x$ ) scripts, respectively.

- Random variables, second order tensors and vectors are represented (63, 77) as functions of the elementary event $\omega$, e.g. $g(\omega), \mathbf{F}(\omega), \boldsymbol{\theta}(\omega)$

- A random field is any function of the spatial coordinates $\boldsymbol{x}$ and the elementary event $\omega$ (e.g. $G(\boldsymbol{x}, \omega))$.

- Fuzzy numbers, vectors, and matrices are denoted by a tilde, like, e.g., $\tilde{m}_{r}$ and $\tilde{\sigma}_{r}$.

- Capital calligraphic letters are used for the domains of functions and sets (e.g. $\mathcal{D}, \mathcal{S}, \mathcal{F}$ ). 
- Bold calligraphic letters denote function spaces like e.g. the Hilbert space $\mathcal{H}$.

- Differential operators are denoted by capital upright letters, e.g. $\mathrm{D}(\boldsymbol{x}, \omega)$.

- In particular Div and Grad denote divergence and gradient operators applied in the reference configuration of a geometrically nonlinear continuous body.

\section{Fuzzy-stochastic finite element analysis}

\subsection{Interval, stochastic and fuzzy analysis}

The discussion in which cases a stochastic (or fuzzy) approach is more advantageous is beyond the scope of this paper. However we should mention that stochastic analysis and fuzzy analysis are alternative in the sense that both may be applied under some assumptions to the same problem, but not equivalent, not concurrent and not competing. These techniques are based on different assumptions, require different input data and provide different output quantities.

In general the stochastic problem description appears naturally for aleatoric uncertainties representing experimentally observed process variability. Aleatoric uncertainties are non-reducible by increasing the experimental effort. No further experimental study will reduce the variation range. The necessary input data is the probability density function. If it is unknown, it may be constructed based on the maximum entropy principle (which is just a convenient assumption) and iteratively improved using, e.g., Bayesian updates.

In contrast epistemic uncertainties are reducible, they result from insufficient knowledge, inaccurate measurements, or roundoff errors. More precise measurements and exact arithmetic could strongly reduce epistemic uncertainty, however, this is not always reasonable. One example where epistemic uncertainty appears and where the probabilistic treatment is not appropriate is error propagation through some algorithm. In this case the researcher is not interested in probabilistic quantities like expectations, but in the interval of confidence only. The task is to find the min and max bounds of the output if the input is defined as interval variable. Obviously interval analysis is the appropriate tool here.

Another example of epistemic uncertainty is considered in this paper. Let us consider some nonlinear physical process, which is studied experimentally. In order to use empirical data in simulations it is fitted with some function. Usually we want to keep the fitting function as simple as possible, we want to avoid overfitting, and further investments in experiments (in order to reduce noise) are not reasonable. Due to the fact that the fitting curve never coincides with experimental data, we want to know, how the changes in the function parameters (different fits) may influence the results. In this case we consider iteratively increasing intervals of confidence for the fitting parameters. The smallest interval of confidence is just a point — the crisp or main value obtained from fitting. The largest interval of confidence (support) is the smallest interval covering all experimental data. The set of nested and ordered intervals results naturally in a fuzzy description. Thus the differences from aleatoric uncertainty in this case are the following: there is no probability distribution and there is no reasoning for its construction; the quantity of 
interest are the intervals of confidence for the output values, but not the expectations; stochastic analysis is highly accurate for averaged values (expectations) but not for the upper and lower bounds estimation — the "worst case scenario"; a fuzzy description appears naturally from the problem formulation.

A third example of epistemic uncertainty, where fuzziness appears, is the case of linguistic variables and approximate reasoning — a common subject in the fuzzy logic community.

Stochastic and fuzzy approaches can be combined for stochastic problems with lack of sufficient input data, thus creating epistemic uncertainty in an otherwise aleatoric setting.

Thus the choice of the approach towards uncertainty is usually dictated by the available information.

Fuzzy and stochastic descriptions are not equivalent. Since the classical work of Zadeh (89) fuzziness is usually interpreted as an application of the possibility theory. The discussion regarding the relation between two approaches is still open. In some cases a fuzzy problem setting may be applied for a stochastic problem as upper and lower bound estimations (84). Possibilities are sometimes interpreted as upper probabilities (20). Or one may just simply replace the pdf by a possibility density of the same form. All these approaches yield different results, also different compared to the stochastic solution. Similarly a fuzzy problem may be replaced by a stochastic problem by assuming some pdf, which is missing in the original setting. Based on the assumed pdf the solution provided will differ strongly.

Furthermore, nowadays there is no unified opinion how to construct and how to understand the possibility density function. In this paper we also address this question and propose one possible interpretation.

\subsection{Stochastic finite element method}

In contrast to usual deterministic FEM the stochastic version works with random parameters thus requiring some preliminary definitions. Let the Euclidean space $\mathbb{E}$ represents the physical space with coordinates $x_{i}$ assembled in the vector $\boldsymbol{x}$. SFEM requires in addition the definition of the stochastic space $\mathbb{S}(25)$. This is the space of random variables with the vector of basic RVs $\boldsymbol{\theta}$. For convenience we choose the basic random variables in form of truncated Gaussian RVs with zero mean and unit variance parameter. The implementation of the truncated Gaussian RVs instead of normal Gaussian RV is motivated by the natural limitations of physical processes (e.g. particle radii cannot tend to infinity in engineering applications) and also for reasons of numerical integration stability $(\underline{64)}$.

All random variables can be described as a nonlinear mapping of the basic set (63, 77). Thus we can visualize the stochastic space $\mathbb{S}$ similar to the physical space $\mathbb{E}$ with coordinates $\theta_{i}(10,11,18,65)$. Thus all other random variables are some functions in $\mathbb{S}$. Please note that the basic RVs are independent, thus their joint probability density function $f_{\Theta}$ represents the product of all individual pdfs from the basic set.

By this definition stochastic FEM may be viewed as normal deterministic FEM, however, in $n$-dimensional physicalstochastic product space $(10,11,18,65)$. 
Galerkin-type FEM as considered in the present work is based on the concept of a Hilbert space of functions. Let us define the physical domain $\mathcal{D} \subset \mathbb{E}$, the stochastic domain $\mathcal{S} \subset \mathbb{S}$, and the tensor product domain $\mathcal{V}=\mathcal{D} \times \mathcal{S}$. Following (25) we introduce the physical Hilbert space $\mathcal{H}$ of functions defined over the domain $\mathcal{D}$, the stochastic Hilbert space $\mathcal{Q}$ of functions defined over the domain $\mathcal{S}$, and the tensor product Hilbert space $\mathcal{W}=\mathcal{H} \times \mathcal{Q}$ of functions defined over the domain $\mathcal{V}$. Physical, stochastic, and product spaces with corresponding domains are depicted in fig. 1
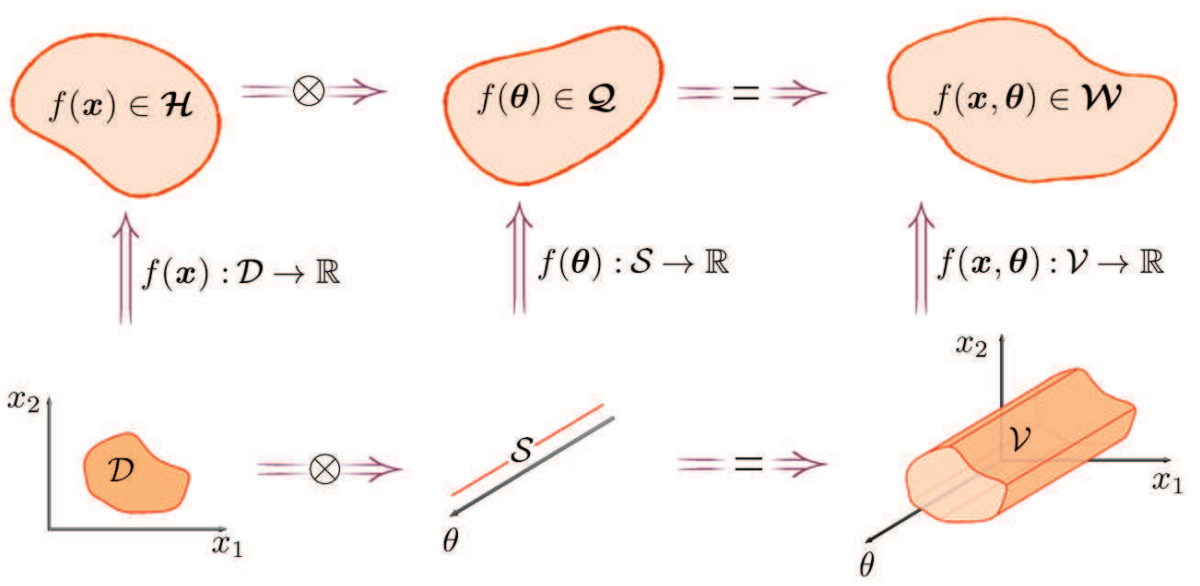

Figure 1: Physical, stochastic, and product function spaces $\mathcal{H}, \mathcal{Q}$, and $\mathcal{W}$ with corresponding physical, stochastic, and product domains $\mathcal{D}, \mathcal{S}$, and $\mathcal{V}$.

SFEM shape functions belong to the space $\mathcal{W}$, thus integration is performed over the domain $\mathcal{V}$.

In this work we use the stochastic local FEM (SL-FEM) approach, which requires unified treatment of physical and stochastic dimensions. Thereby local quadratic $n$-dimensional serendipity-type shape functions (65) are used for the discretization of the domain $\mathcal{V}$.

Let \langle\rangle denote the inner product in the physical-stochastic product space.

$$
\left\langle g_{1}(\boldsymbol{x}, \omega) g_{2}(\boldsymbol{x}, \omega)\right\rangle:=\int_{\mathcal{D}} \int_{\mathcal{S}} g_{1}(\boldsymbol{x}, \boldsymbol{\theta}) g_{2}(\boldsymbol{x}, \boldsymbol{\theta}) \mathrm{d} \boldsymbol{x} f_{\Theta} \mathrm{d} \boldsymbol{\theta} .
$$

Next we consider a random differential operator $\mathrm{D}(\boldsymbol{x}, \omega)$ such that

$$
\mathrm{D}(\boldsymbol{x}, \omega) \boldsymbol{y}(\boldsymbol{x}, \omega)=f(\boldsymbol{x}, \omega) .
$$

where $f(\boldsymbol{x}, \omega)$ is the random loading and $\boldsymbol{y}(\boldsymbol{x}, \omega)$ is the unknown function. 
Thus, Galerkin projections of the differential operator $\mathrm{D}(\boldsymbol{x}, \omega)$ and the unknown function $\boldsymbol{y}(\boldsymbol{x}, \omega)$ onto the basis $\boldsymbol{\varphi}(\boldsymbol{x}, \omega)$ yield

$$
\begin{gathered}
\boldsymbol{y}(\boldsymbol{x}, \omega)=\sum_{i=1}^{\infty} \boldsymbol{y}_{i} \varphi_{i}(\boldsymbol{x}, \omega), \\
\left\langle[\mathrm{D}(\boldsymbol{x}, \omega) \boldsymbol{y}(\boldsymbol{x}, \omega)-f(\boldsymbol{x}, \omega)] \varphi_{i}(\boldsymbol{x}, \omega)\right\rangle=0, \\
\forall i=1, \ldots, N .
\end{gathered}
$$

where $N$ is the number of basis functions.

For a nonlinear mechanical problem the differential operator in (2) reads

$$
\begin{aligned}
& \mathrm{D}(\boldsymbol{x}, \omega) \boldsymbol{y}(\boldsymbol{x}, \omega):=-\operatorname{Div} \mathbf{P}(\mathbf{F}(\boldsymbol{x}, \omega)), \\
& \mathbf{F}=\operatorname{Grad} \boldsymbol{y}(\boldsymbol{x}, \omega), \\
& f(\boldsymbol{x}, \omega):=\boldsymbol{f}(\boldsymbol{x}, \omega),
\end{aligned}
$$

where $\boldsymbol{y}(\boldsymbol{x}, \omega)$ corresponds to the random deformation map describing the position of material points in the actual configuration, $\boldsymbol{f}(\boldsymbol{x}, \omega)$ denotes the random body forces, $\mathbf{P}$ and $\mathbf{F}$ represent the Piola stress tensor and the deformation gradient tensor, respectively. Please note that the Grad and Div operators involve differentiation only with respect to the physical coordinates $\boldsymbol{x}$ in the material configuration.

For the sake of demonstration the Piola stress tensor $\mathbf{P}(\mathbf{F}(\boldsymbol{x}, \omega))$ is given as the first derivative of the Neo-Hookean energy potential $\Psi(\mathbf{F})$.

$$
\begin{gathered}
\Psi(\mathbf{F})=\frac{1}{2} \Lambda[\mathbf{F}: \mathbf{F}-3-2 \ln J]+\frac{1}{2} \lambda \ln ^{2} J \\
\frac{\partial \Psi}{\partial \mathbf{F}}=\mathbf{P}=\Lambda \mathbf{F}+\lambda_{0} \mathbf{F}^{-t},
\end{gathered}
$$

where $\Lambda$ and $\lambda$ are Lamé parameters, with $\Lambda$ the shear modulus and $\lambda$ is related to Poisson's ratio $\nu=\frac{\lambda}{2[\lambda+\Lambda]} ; \lambda_{0}=[\lambda \ln J-\Lambda]$; $\mathbf{C}^{-1}$ is the inverse of the right Cauchy-Green tensor $\mathbf{C}=\mathbf{F}^{t} \cdot \mathbf{F} ; J=\operatorname{det} \mathbf{F}$ is the Jacobian determinant; $\mathbf{F}^{t}$ denotes the transpose of $\mathbf{F}$ and $\mathbf{F}^{-t}$ denotes the transposed inverse of $\mathbf{F}$. 
Thus, the expressions (4) and (5) after integration by parts read

$$
\begin{aligned}
& \boldsymbol{R}=\boldsymbol{F}^{\mathrm{ext}}-\boldsymbol{F}^{\mathrm{int}} \rightarrow \boldsymbol{O}, \\
& \boldsymbol{F}_{i}^{\mathrm{int}}=\left\langle\mathbf{P} \cdot \operatorname{Grad} \varphi_{i}(\boldsymbol{x}, \omega)\right\rangle, \\
& \boldsymbol{F}_{i}^{\mathrm{ext}}=\left\langle\boldsymbol{f}(\boldsymbol{x}, \omega) \varphi_{i}(\boldsymbol{x}, \omega)\right\rangle,
\end{aligned}
$$

where $\boldsymbol{R}$ is the residual and \langle\rangle is the inner product in $\mathcal{W}$. Here for simplicity of exposition only the Dirichlet problem is considered.

Newton iterations are used in order to find the solution.

$$
\begin{gathered}
\boldsymbol{R}^{k}(\boldsymbol{Y})+\left.\frac{\partial \boldsymbol{R}^{k}}{\partial \boldsymbol{Y}}\right|_{\boldsymbol{Y}} \cdot \mathrm{d} \boldsymbol{Y}^{k} \rightarrow \boldsymbol{O} \\
\mathbf{K}^{k}:=-\left.\frac{\partial \boldsymbol{R}^{k}}{\partial \boldsymbol{Y}}\right|_{\boldsymbol{Y}}, \\
\mathrm{d} \boldsymbol{Y}^{k}=\left[\mathbf{K}^{k}\right]^{-1} \boldsymbol{R}^{k} \\
\boldsymbol{Y}^{k+1}=\boldsymbol{Y}^{k}+\mathrm{d} \boldsymbol{Y}^{k}
\end{gathered}
$$

Here $\boldsymbol{Y}$ is a vector of coefficients in the finite approximation of (3)

$$
\boldsymbol{Y}=\left[\begin{array}{c}
\boldsymbol{y}_{1} \\
\boldsymbol{y}_{2} \\
\vdots \\
\boldsymbol{y}_{n}
\end{array}\right], \quad \boldsymbol{y}_{i}=\left[\begin{array}{c}
y_{i 1} \\
y_{i 2} \\
\vdots
\end{array}\right],
$$

where $y_{i j}$ represents the $j$-th component of the solution field projected onto the $i$-th basis function.

The explicit expression for the stiffness matrix $\mathbf{K}$ reads

$$
\mathbf{K}_{i j}=\left\langle\frac{\partial \mathbf{P}}{\partial \mathbf{F}} \overline{:}\left[\operatorname{Grad} \varphi_{i}(\boldsymbol{x}, \omega) \otimes \operatorname{Grad} \varphi_{j}(\boldsymbol{x}, \omega)\right]\right\rangle
$$


with

$$
\frac{\partial \mathbf{P}}{\partial \mathbf{F}}=\frac{\partial^{2} \Psi}{\partial \mathbf{F}^{2}}=\lambda \mathbf{F}^{-t} \otimes \mathbf{F}^{-t}-\lambda_{0} \mathbf{F}^{-t} \underline{\otimes} \mathbf{F}^{-1}+\Lambda \mathbf{I} \otimes \mathbf{I}
$$

where $\mathbf{I}$ is the identity tensor; $:$ denotes the non-standard double contraction of a fourth order tensor $\mathbf{A}$ and a second order tensor $\mathbf{B}$ represented component-wise by $[\mathbf{A}: \mathbf{B}]_{i k}=[\mathbf{A}]_{i j k l}[\mathbf{B}]_{j l}$; the symbols $\bar{\otimes}$ and $\underline{\otimes}$ denote the non-standard tensor products of two second order tensors $\mathbf{A}$ and $\mathbf{B}$ represented component-wise as follows: $[\mathbf{A} \bar{\otimes} \mathbf{B}]_{i j k l}=[\mathbf{A}]_{i k}[\mathbf{B}]_{j l}$ and $[\mathbf{A} \underline{\otimes \mathbf{B}}]_{i j k l}=$ $[\mathbf{A}]_{i l}[\mathbf{B}]_{j k}$.

Please note that the gradients of the basis functions are derived in the $n$-dimensional product space with coordinates $\{\boldsymbol{x}, \boldsymbol{\theta}(\omega)\}$, whereas the position vector has only three elements corresponding to the physical coordinates $\boldsymbol{x}$. Observe that the model exhibits no deformation in stochastic directions.

\subsection{Fuzzy finite element analysis}

In many cases the system parameters cannot be obtained exactly due to many reasons: lack of knowledge regarding the microstructure, limited accuracy of the fitting procedure, imperfection of the model, which can be fitted to the experimental data but never coincides with them, noisy experimental data, insufficient number of experimental samples, etc. In these cases no statistical data for system parameters is provided. Thus the probabilistic description of the unknown parameters is getting problematic. In this case a highly efficient tool for the forward propagation of epistemic uncertainty is fuzzy arithmetic (30).

Here, the uncertain parameters are represented not in terms of random variables but in terms of fuzzy numbers.

The history of fuzzy number began in 1965 with the introduction of fuzzy sets (90), which are an extension of the classical set theory based on the notion of different grades of membership. In the case of a fuzzy set $\tilde{\mathcal{X}}$ the grade of membership of $x$ is defined by the membership function $\mu_{\tilde{\mathcal{X}}}(x) \in[0,1]$. Here $\mu_{\tilde{\mathcal{X}}}(x)=1$ means that the element $x$ entirely belongs to the set $\tilde{\mathcal{X}}$, $\mu_{\tilde{\mathcal{X}}}(x)=0$ means that $x$ is definitely not a member of the set $\tilde{\mathcal{X}}$. In the case of a conventional set $\mathcal{X}$ the membership function of some element $x$ may have only two values $\mu_{\mathcal{X}}(x) \in\{0,1\}$, i.e. the element can only entirely belong to or not belong to the set $\mathcal{X}$.

For practical applications a few very important types of fuzzy sets are fuzzy numbers, fuzzy intervals, crisp numbers, and crisp intervals. A fuzzy number $\tilde{a}$ is the convex fuzzy set over the universal set $\mathbb{R}$ with the membership function $\mu_{\tilde{a}}(x) \in[0,1]$, where $\mu_{\tilde{a}}(x)=1$ only for one single value of $x=\bar{a}$ called the modal value. The fuzzy interval $\tilde{A}$ is the convex fuzzy set defined similarly to the fuzzy number, however with the difference that $\mu_{\tilde{A}}(x)=1$ holds for some interval called modal interval $\bar{A}$. A crisp interval $A$ can be considered as the fuzzy set of points such that $\mu_{A}(x)=1$, if $x \in A$, and $\mu_{A}(x)=0$ otherwise. The crisp number $a$ is then the fuzzy set with the membership function given by the Kronecker delta function $\mu_{a}(x)=\delta(x, a)$. Fig. 2 represents from left to right: crisp number $x=1$, crisp interval $[2,3]$, symmetric triangular fuzzy number with $\bar{x}=4.5$, fuzzy 
interval with the modal interval $x \in[6.5,7.5]$, and the arbitrary non-convex subnormal fuzzy set with nonzero membership function on the interval $x \in[9,11]$.

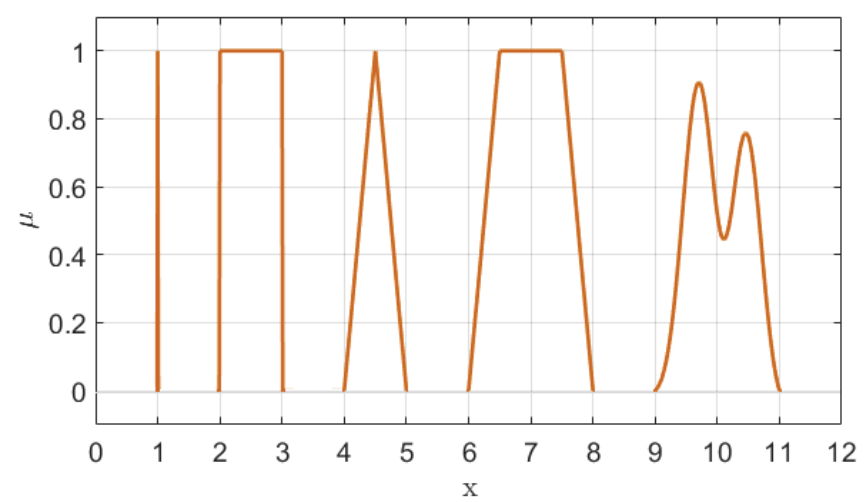

Figure 2: Membership function plotted for (from left to right): crisp number, crisp interval, symmetric triangular fuzzy number, fuzzy interval, and the arbitrary non-convex subnormal fuzzy set.

Zadeh's extension principle is used to perform unary and binary arithmetical operations of fuzzy numbers. For any arbitrary function $f$ applied to the pair of fuzzy numbers $\tilde{a}$ and $\tilde{b}$ the membership function of the resulting fuzzy number $\tilde{c}=f(\tilde{a}, \tilde{b})$ reads

$$
\mu_{\tilde{c}}(z)=\sup _{z=f(x, y)} \min \left\{\mu_{\tilde{a}}(x), \mu_{\tilde{b}}(y)\right\}
$$

Due to the high complexity of calculations performed using the extension principle an alternative approach was proposed in the literature. The fuzzy numbers are reduced to sets of intervals for different degrees of membership, i.e. $\alpha$-cuts. These intervals are also called intervals of confidence (30). Thus for every $\alpha$-cut interval arithmetic can be applied. Fig. 3 represents a triangular fuzzy number decomposed into six $\alpha$-cuts. For $\alpha$-cut $\mu(x)=1$ the problem is reduced to a simple deterministic one. For all other $\alpha$-cuts the interval problem is considered. In practical application the interval problem is reduced to two optimization problems (global min and max on interval) at each $\alpha$-level to obtain the correct bounds of the output interval. The goal function here is the quantity of interest. The uncertain parameters are the design variables.

However, if the evaluation of the system is costly, the optimization approach becomes too expensive. As an alternative one may use the extended transformation method (30). Thus we obtain an approximate solution using a limited predefined number of samples, which may be generated using full grid or sparse grid techniques. In this work we focus on the standard full grid approach due to its stability and accuracy.

The structure of the proposed fuzzy-stochastic homogenization framework is as follows. Based on the experimental data we design a stochastic RVE. Parametrized distributions like, e.g., truncated Gaussian or truncated log-normal, are used to fit 


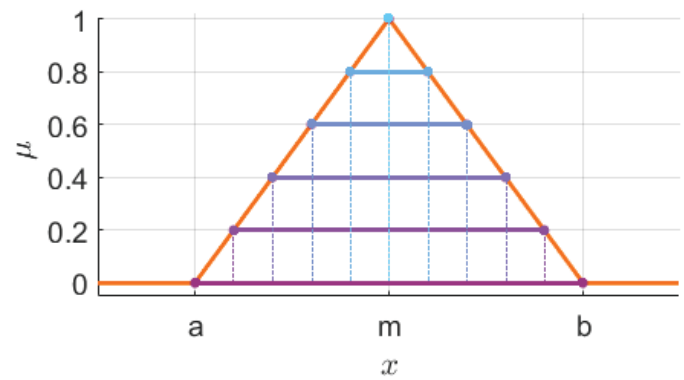

Figure 3: Triangular fuzzy number with modal value $m_{x}$ decomposed into $6 \alpha$-cuts.

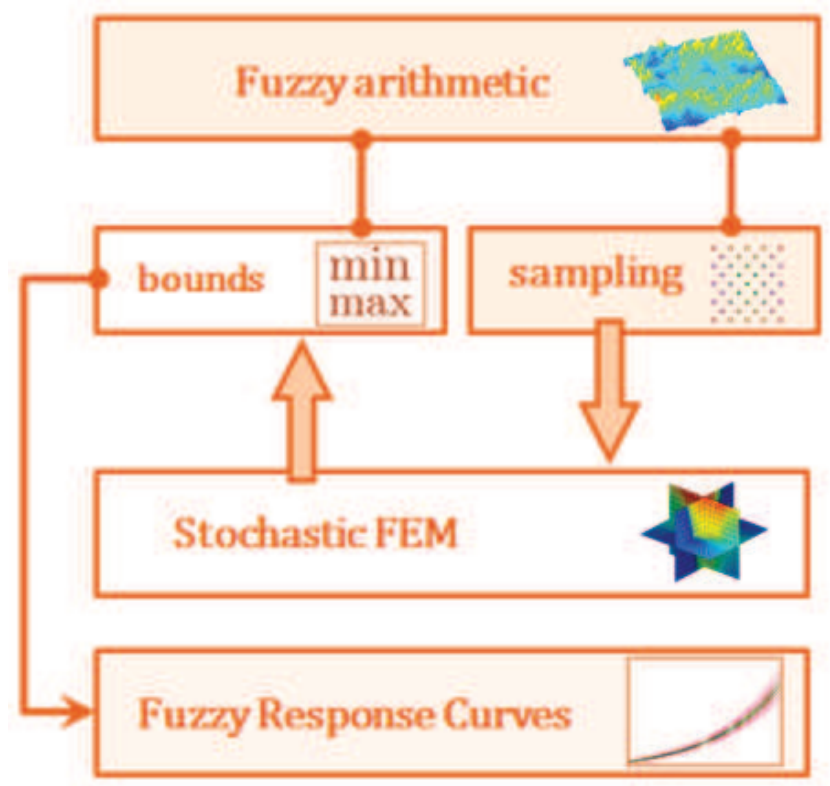

Figure 4: Structure of the fuzzy-stochastic FEM based homogenization framework.

statistical data. The distribution parameters cannot be estimated exactly thus becoming fuzzy numbers. Full grid sampling is used. Thus every sample is resolved using the isoparametric stochastic local FEM with unified discretization in the physical and the stochastic domains. The SFEM output is then analyzed in order to construct the response surfaces for every quantity of interest, e.g. for the homogenized stress mean value. Response surfaces are used to extract min and max values of the quantities of interest for every $\alpha$-cut. Finally fuzzy response curves are plotted representing upper and lower bounds for every $\alpha$-cut. The block-scheme of the presented algorithm is depicted in fig. 4

\subsection{Random variable transformation}

Any random variable in $\mathcal{Q}$ can be represented as some function of the basic random variables. For the application of the SFEM technique we need representation of all random variables in terms of basic RVs. For some standard RV models like, e.g., log-normal, the representation in terms of Gaussian RV is well-known. For other RVs, especially for those, which describe 
experimental data or simulation output, this kind of representation should be defined. Moreover we use this mapping to generate random samples with arbitrary distribution.

In the case of random inclusion radii the space $\mathbb{S}$ is one-dimensional with only one basic RV $\theta(\omega)$, which is chosen in the form of a truncated Gaussian RV. This truncation is discussed and motivated in $(64,65)$.

Thus any random radius $r(\omega)$ with pdf $f_{r}(r(\omega))$ can be presented as a function $r(\omega)=r(\theta(\omega))$. This mapping may be nonlinear and non-unique. Thus the computation of the function $r(\theta(\omega))$ becomes non-trivial (63, 77). However, if we consider a monotonically increasing function $r(\theta(\omega))$, the following relation holds (63)

$$
\mathrm{d} r f_{r}(r)=\mathrm{d} \theta f_{\theta}(\theta)
$$

Thereby we introduce a unique mapping of the points in the support of $\theta(\omega)$ into the points in the support of $r(\omega)$. Relation (14) states that the probability mass at every point stays unchanged during this mapping, which is in fact the probability mass conservation law.

The mapping function is computed as the solution of the differential equation

$$
\frac{\mathrm{d} r}{\mathrm{~d} \theta}=\frac{f_{\theta}(\theta)}{f_{r}(r)}
$$

Thereby the pdfs should satisfy the following requirements:

- all pdfs should be at least $C^{0}$-continuous,

- all RVs should posses finite support,

- $f_{r}(r)>0$ for all $r$ in the support.

Mapping of a random variable with infinite support into a RV with finite support and vice versa is also possible, however the mapping function should be singular at the ends of the interval thus increasing the problem's complexity. The ODE 15 is complemented with two boundary conditions stating that the max and min values of one RV are mapped one-to-one into the max and min values of another RV, respectively. The second boundary condition may be used for accuracy control.

Due to the fact that $f_{\theta}$ and $f_{r}$ are close to zero at the ends of the interval, numerical solution of (15) may be inaccurate or unstable. Moreover the obtained curves may be close to singular. Thus we transform the problem to the form

$$
\begin{aligned}
& \frac{\mathrm{d} t}{\mathrm{~d} r}=f_{r}(r), \\
& \frac{\mathrm{d} t}{\mathrm{~d} \theta}=f_{\theta}(\theta), \\
& \forall t \in[0,1]: f_{t}(t)=1 .
\end{aligned}
$$


Both equations can be solved using the Runge-Kutta method. The solutions obtained are tabulated functions $t(r)$ and $t(\theta)$ which can be transformed to $r(t)$ and $\theta(t)$ just by swapping columns. Thereby we obtain the curve $r(\theta)$ in parametric form. This method is also suitable for almost singular curves.

Expression (14) is also often used to obtain the unknown function $f_{r}(r)$, if $f_{\theta}(\theta)$ and $r(\theta)$ are given:

$$
f_{r}(r(\theta))=f_{\theta}(\theta)\left[\frac{\mathrm{d} r}{\mathrm{~d} \theta}(\theta)\right]^{-1}
$$

\section{Stochastic representative volume element}

Homogenization considers typically two separate scales: the macro scale and the micro scale. Thereby macroscopic material properties are obtained from the simulation of the microscopic model. In the case of random material microstructures the microscopic model should be large enough to exhibit all macroscopic properties, thus resulting in extremely high computational costs. Thus the ergodic assumption is often used, which states that the averaging over one large sample is equivalent to the averaging over the ensemble of small samples. Thereby time and computer power demanding simulations can be replaced by the analysis of one small stochastic RVE, however, including statistical information about the microstructural variability.

We base our RVE design on the experimental data and scanning electron microscopy (SEM) pictures of iron particle filled elastomers established at the Chair of Applied Mechanics, University of Erlangen-Nuremberg (fig. 5).

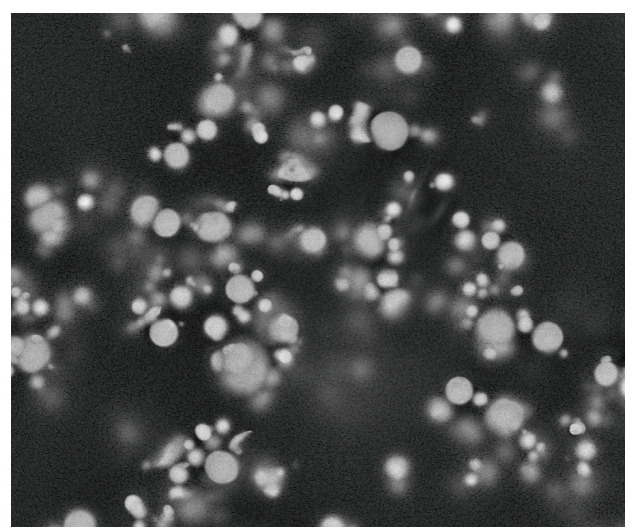

Figure 5: Scanning electron microscopy of an iron particle filled elastomer (Courtesy of Bastian Walter, Chair of Applied Mechanics, University of Erlangen-Nuremberg)

Following our previous works we consider a rectangular RVE with the size $2 a$, where $a=1$, with one circular inclusion possessing random radius $r(\omega)$. Due to the periodic boundary conditions the inclusions position inside the RVE does not influence the homogenized stress values, thus for the sake of simplicity we consider only the model with the inclusion in the center of the RVE. 
The stochastic RVE can be imagined for the simplest case (two physical and one stochastic coordinates) as a stack of thin sheets with deterministic 2D RVEs in each of them, i.e. every horizontal slice of the stochastic RVE corresponds to some deterministic model. Thus the vertical dimension demonstrates the evolution of the microstructure by varying the random parameter (fig. 6).

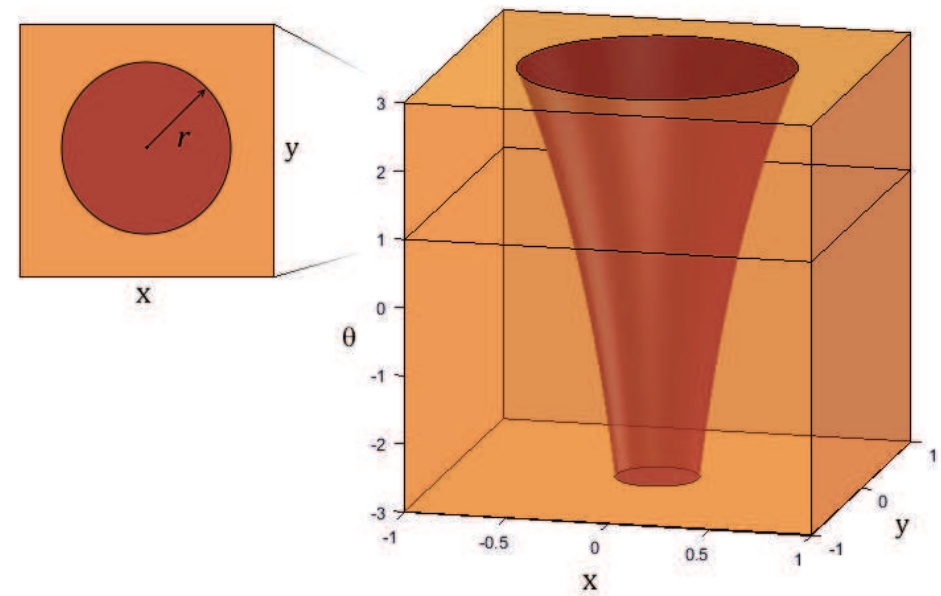

Figure 6: The stochastic representative volume element for a 2D problem considering the random inclusion's radius.

We model an inclusion as a jump in elastic properties $\left(C^{-1}\right.$-continuity), whereby the displacements are $C^{0}$-continuous. We assume for simplicity a constant Poisson's ratio $\nu=0.3$. In the general case the Poisson's ratio is also a random field. An interesting analysis for the case of fluctuating Poisson's ratio is presented, e.g. in (38).

Thus only the shear modulus is a random field and is given as

$$
G(\chi)=G_{m}+\frac{1}{2}\left[G_{i}-G_{m}\right][1-\operatorname{sign} z(\boldsymbol{x}, \omega)]
$$

where $G_{m}$ and $G_{i}$ are the shear moduli of the matrix and the inclusion, respectively; $z(\boldsymbol{x}, \omega)$ is a cone-like level-set function (64), which indicates whether the material point with coordinates $x$ belongs to the matrix or to the inclusion $(z<0$ : inclusion, $z>0$ : matrix)

$$
z(\boldsymbol{x}, \omega)=r(\omega)\left[\sqrt{\frac{x_{1}^{2}}{r(\omega)^{2}}+\frac{x_{2}^{2}}{r(\omega)^{2}}}-1\right] .
$$

The random radius $r(\omega)$ should be represented as the mapping of the basic random variable $\theta(\omega)$. Determination of the experimentally motivated models of $r(\theta(\omega))$ are presented in the sequel. In this work we propose two models describing the 
random radius of the inclusion. The first model is motivated by the experimental study of the inclusions' radii. The second model uses also the information about the inclusions' distribution to determine the size of the RVE.

In order to verify our model, we use the values of material parameters from (34): $G_{m}=8, G_{i}=80$.

All simulation were performed with periodic boundary conditions applied to the boundaries of the RVE. The macroscopic loading is presented by the macroscopic deformation gradient $\overline{\mathbf{F}}$ describing a $10 \%$ uniaxial stretch:

$$
\overline{\mathbf{F}} \widehat{=}\left[\begin{array}{ccc}
1.1 & 0 & 0 \\
0 & 1 & 0 \\
0 & 0 & 1
\end{array}\right] .
$$

\subsection{Stochastic RVE with fixed cell size}

The first model of the particle radii distribution considers a fixed size of the RVE. This model is motivated by the experimental study of the inclusions' radii presented in fig. 7. The experimentally obtained radii distribution exhibits a behavior very close to log-normal. Typically the log-normal random parameters are represented as a nonlinear mapping of the basic Gaussian RV.

$$
r(\theta(\omega))=\exp \left(m_{r}+\sigma_{r} \theta(\omega)\right)
$$

where $m_{r}$ and $\sigma_{r}$ are location and scale parameters of the log-normal distribution, respectively.

The least square fitting procedure is used to evaluate the parameters of the log-normal distribution. Thereby we fit the cumulative distribution function (cdf) provided in fig. 7 Note that the histogram presenting the pdf of the radii distribution is not suitable for the parameter fitting due to the lack of information about the bins width. Moreover, while evaluating the cdf, the influence of the noise on measurements is partially compensated by the averaging procedure. Thus the experimental cdf is usually more accurate compared to the experimental pdf.

In order to perform parameter fitting, we normalize the radius values in the experimental curve thus changing to some dimensionless radius $r_{n}$ which represents the ratio of the radius to the RVE size. In this work we consider the size of the RVE $a=10 \mu m$. Thus the RVE with $a=10 \mu m$ is large enough to contain more than $99.7 \%$ of all considered inclusions radii. Note that fig. 7 demonstrates the cdf plotted versus the diameter thus the inclusion with diameter $20 \mu m$ (purple vertical line in fig. 7) completely fits into the RVE with size $a=10 \mu \mathrm{m}$. 


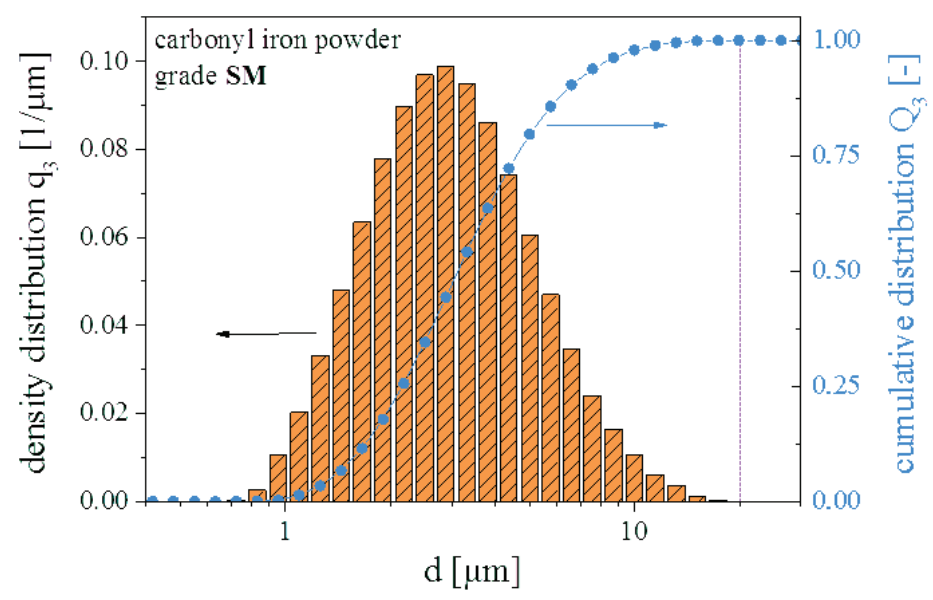

Figure 7: Histogram of inclusion diameter distribution and the obtained cumulative probability density (Courtesy of Bastian Walter, Chair of Applied Mechanics, University of Erlangen-Nuremberg).

The fitting problem is formulated as follows:

$$
\begin{aligned}
& \left\{\bar{m}_{r}, \bar{\sigma}_{r}\right\}=\arg \min _{m, \sigma}\left\|F_{(m, \sigma)}\left(r_{n}\right)-F_{\exp }\left(r_{n}\right)\right\|_{L^{2}}, \\
& \sigma_{r}>0
\end{aligned}
$$

where $\|\cdot\|_{L^{2}}$ is the $L^{2}$-norm, $F_{e x p}\left(r_{n}\right)$ and $F_{(m, \sigma)}\left(r_{n}\right)$ are the experimental cdf and the log-normal cdf with parameters $m$ and $\sigma$, respectively.

The inclusions' volume fraction $v$ is evaluated for the considered cell size through the obtained log-normal pdf:

$$
\begin{aligned}
& v=\frac{\pi}{4} \int_{0}^{1} r_{n}^{2} f_{\left(\bar{m}_{r}, \bar{\sigma}_{r}\right)}\left(r_{n}\right) \mathrm{d} r_{n}, \\
& f_{\left(\bar{m}_{r}, \bar{\sigma}_{r}\right)}\left(r_{n}\right)=\frac{\mathrm{d} F_{\left(\bar{m}_{r}, \bar{\sigma}_{r}\right)}}{\mathrm{d} r_{n}}\left(r_{n}\right) .
\end{aligned}
$$

For the cell size $a=10 \mu m$ the volume fraction is just $v=3.64 \%$.

Fig. 8 demonstrates the fitting curve plotted over experimental data. Note the slight disagreement between the log-normal distribution and the empirical curve. Due to the fact that exact fitting of the experimental data is impossible, the model parameters obtained cannot be considered as exact values, but rather represent fuzzy numbers $\tilde{m}_{r}$ and $\tilde{\sigma}_{r}$. Correspondingly the fuzzy $\log$-normal cdf $F_{(\tilde{m}, \tilde{\sigma})}\left(r_{n}\right)$ is introduced. Black dashed lines in fig. 8 represent upper and lower bounds of the fuzzy log-normal distribution containing the entire experimental data.

The procedure of reconstructing the fuzzy model parameters based on experimental data is as follows:

- The obtained parameter values $\bar{m}_{r}$ and $\bar{\sigma}_{r}$ representing the best fit in the sense of least squares are considered to be the fuzzy parameter's modal values. 
- Some variations of the parameter's values $\Delta m_{1}, \Delta m_{2}, \Delta \sigma_{1}$, and $\Delta \sigma_{2}$ are introduced into the model.

- Due to the parameter variations the log-normal cdf is represented not by a single curve but rather by upper and lower bounds $F_{\min }(r)$ and $F_{\max }(r)$.

- The experimental data exceeding the region between $F_{\min }(r)$ and $F_{\max }(r)$ is denoted as residual.

- The optimization problem is solved, wherein the $\Delta m_{1}, \Delta m_{2}, \Delta \sigma_{1}$, and $\Delta \sigma_{2}$ are design variables, the residual is the goal function to be minimized.

- Based on the modal values $\bar{m}_{r}$ and $\bar{\sigma}_{r}$ and the obtained variations $\Delta m_{1}, \Delta m_{2}, \Delta \sigma_{1}$, and $\Delta \sigma_{2}$ we reconstruct asymmetric triangular fuzzy parameters $\tilde{m}_{r}$ and $\tilde{\sigma}_{r}$.

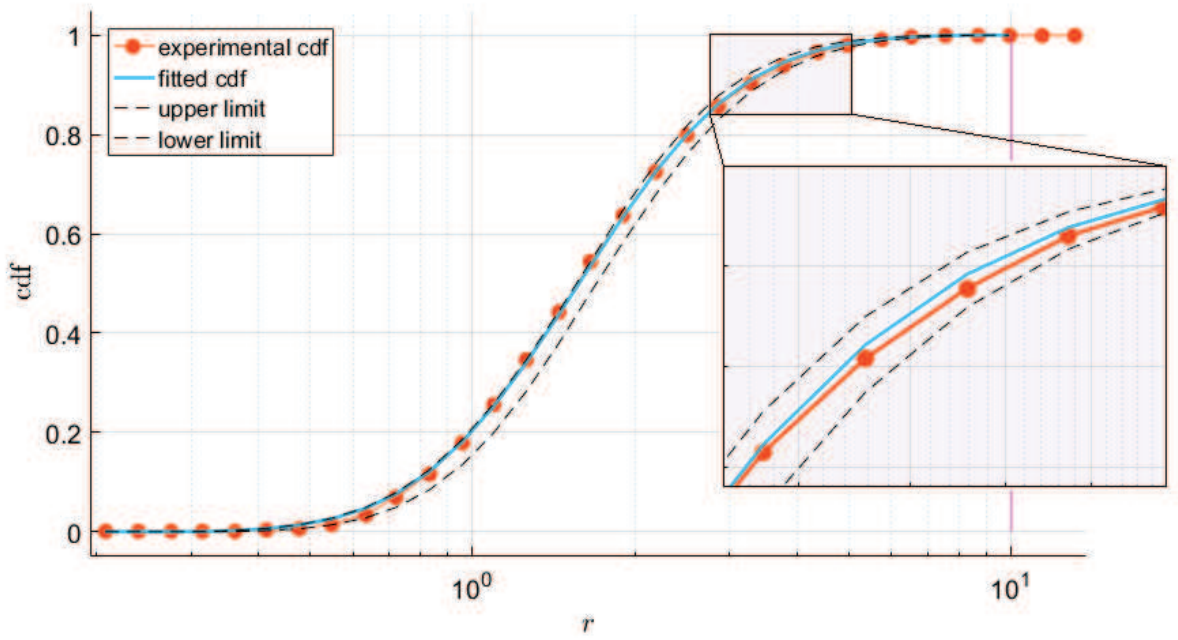

Figure 8: Log-normal fitting of the experimental curve representing the cdf of the inclusions radii.

In order to estimate upper and lower bounds for the given variations of $m_{r}$ and $\sigma_{r}$ we introduce the following triplets.

$$
\begin{aligned}
& m_{r} \in\left\{\bar{m}_{r}, \bar{m}_{r}-\Delta m_{1}, \bar{m}_{r}+\Delta m_{2}\right\}, \\
& \sigma_{r} \in\left\{\bar{\sigma}_{r}, \bar{\sigma}_{r}-\Delta \sigma_{1}, \bar{\sigma}_{r}+\Delta \sigma_{2}\right\},
\end{aligned}
$$

Thus we obtain nine pairs of parameters $m_{r}$ and $\sigma_{r}$ representing nine curves $r_{i j}(\theta(\omega))=\exp \left(m_{r i}+\sigma_{r j} \theta(\omega)\right)$ with corresponding cumulative distribution functions $F_{i j}\left(r_{n}\right)$.

Upper and lower bounds are defined as

$$
\begin{aligned}
& F_{\max }\left(r_{n}\right)=\max _{i j}\left(F_{i j}\left(r_{n}\right)\right), \\
& F_{\min }\left(r_{n}\right)=\min _{i j}\left(F_{i j}\left(r_{n}\right)\right) .
\end{aligned}
$$


For the given upper and lower bounds the residual curve represents the experimental data exceeding the region between $F_{\min }(r)$ and $F_{\max }(r)$ schematically shown in fig. 9

$$
\begin{aligned}
& R\left(r_{n}\right)=R_{\max }\left(r_{n}\right)+R_{\min }\left(r_{n}\right), \\
& R_{\max }\left(r_{n}\right)=\max \left\{F_{\text {exp }}\left(r_{n}\right)-F_{\max }\left(r_{n}\right), 0\right\}, \\
& R_{\min }\left(r_{n}\right)=\min \left\{F_{\min }\left(r_{n}\right)-F_{\text {exp }}\left(r_{n}\right), 0\right\} .
\end{aligned}
$$

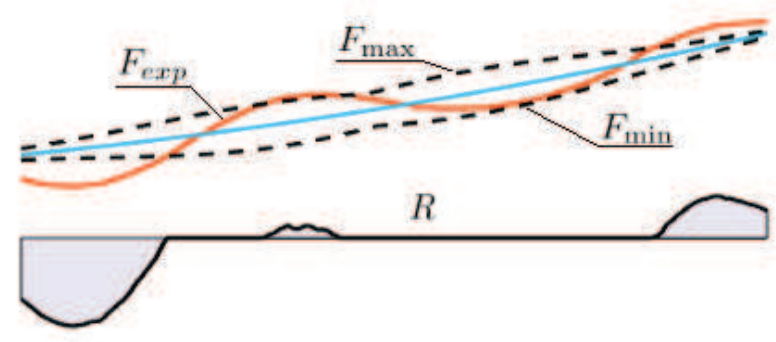

Figure 9: Experimental cdf $\left(F_{\text {exp }}\right)$, upper $\left(F_{\max }\right)$ and lower $\left(F_{\min }\right)$ bounds of the theoretical cdf and the residual curve $(R)$ obtained according to (25).

Lower and upper bounds are obtained as solution of the optimization problem with four design variables, i.e. parameter variations $\Delta=\left[\Delta m_{1}, \Delta m_{2}, \Delta \sigma_{1}, \Delta \sigma_{2}\right]$. The optimization problem is defined as

$$
\begin{aligned}
& \Delta=\arg \min _{\Delta}\left(W_{1}\|\Delta\|_{L^{2}}+W_{2}\left\|R\left(r_{n}, \Delta\right)\right\|_{L^{2}}\right), \\
& W_{1}=\left[\bar{m}_{r}^{2}+\bar{\sigma}_{r}^{2}\right]^{-1}, \\
& W_{2}=\left\|F_{\left(\bar{m}_{r}, \bar{\sigma}_{r}\right)}\left(r_{n}\right)-F_{\text {exp }}\left(r_{n}\right)\right\|_{L^{2}}^{-1}, \\
& \Delta_{i}>0 .
\end{aligned}
$$

Thus we minimize the length of the vector $\Delta$ containing positive parameter variations together with the penalty term for experimental data exceeding the theoretical boundaries. The vector of design variables and the penalty term are normalized with respect to the parameter's modal values (21) and square-norm of the difference between the experimental curve and the log-normal cdf, respectively.

Thus for both uncertain parameters we obtain left and right bounds. Accordingly we introduce two asymmetric triangular fuzzy numbers: $\tilde{m}_{r}$ with modal value $\bar{m}_{r}=-1.8475$ defined in the range $[-1.8565,-1.7745]$ and $\tilde{\sigma}_{r}$ with modal value $\bar{\sigma}_{r}=0.5466$ defined in the range $[0.5206,0.5495]$.

Due to the fuzzy-random nature of the radius $r_{n}$ the elastic properties of matrix and inclusion (18) are represented as fuzzyrandom fields necessitating the use of the fuzzy-stochastic finite element method. 


\subsection{Stochastic RVE with variable cell size}

An attempt to describe the inclusions' positions in terms of RVs results in a large and complex system of RVs with strongly nonlinear dependencies between them. Therefore some alternative approach should be established. So, e.g., in (24) the authors used the probabilistic description of the interparticle distances, which was assumed known. Thereby the authors consider only one particle (center) surrounded by an infinite set of particles with random distances to the center. The disadvantage of this approach is that the interparticle distances between the surrounding particles are not considered. Moreover the introduction of any restrictions on the distances between the surrounding particles require the use of three-, four-, five-, and $n$-point distribution density functions thus resulting in a system of the same complexity as the model with random positions.

Alternatively one may focus instead of interparticle distances on the free area around the inclusion associated with this inclusion, thus, creating an RVE with variable size. Thereby we propose to use a statistically similar representative volume element (75). The idea is to provide some substitute or surrogate model, which possesses some statistical properties of the original model, specifically the relation between the inclusion's radius and the area around the inclusion. The statistically similar stochastic model is simple and contains only one inclusion. By varying the inclusion's radii distribution in a one-inclusion model we may control not only the average stress, but also the maximum and minimum stress and the stress standard deviation in the stochastic system.

Please note that we seek for an equivalence not between two deterministic models but between a deterministic model with randomly distributed inclusions and a stochastic model with one single inclusion. Thus we utilize the ergodicity concept (23, 24). The idea is to develop a stochastic model (statistically similar, simple, and optionally periodic) whereby the average over ensembles is assumed equivalent to the average over the volume of the deterministic model with randomly distributed inclusions (fig. 10).

The use of periodic boundary conditions for the statistically similar (substitute) model is motivated by a number of studies demonstrating that the periodic boundary conditions are the most reliable and converge faster than Dirichlet and Neumann boundary conditions $(69,70,87)$. They are often used even if the model is not periodic $(70,87)$, because Dirichlet and Neumann boundary conditions give always overestimation and underestimation for the stress $(69,70,73,87)$ in computational homogenization. Moreover the simple comparison of the homogenized stresses in periodic composites modeled with only one inclusion in the unite cell and composites with random microstructure, performed in ( 187$)$, demonstrated close homogenized stress values in both models. Thus, one of the conclusions made in $(87)$ is that one unit cell with only one centered inclusion and periodic boundary conditions is already a good approximation for the model with randomly distributed particles at least in engineering applications.

All necessary statistical information concerning the free area distribution may be estimated statistically from the experimental data.

The design of an RVE with variable cell size includes the following steps: 
- Real materials possess randomly distributed non-overlapping inclusions. One may divide microscopic material samples into cells wherein every cell represents the set of material points that are closer in some sense to the associated inclusion than to any other.

. The cell area distribution can be estimated statistically based on the analysis of microscopic samples.

- We consider a rectangular RVE with size parameter $a$ and total area $A=4 a^{2}$. The area of the RVE is considered to exhibit the same distribution density function as the areas of the cells.

. The influence of the cell's shape is not considered.

- The influence of the particle position within the RVE is neglected by using periodic boundary conditions.

- The number of model parameters is reduced by introducing the normalized (reduced) radius $r_{n}=r / a$. Thus all statistical information regarding the radius variation and cell area distribution is included in the probability density function of the normalized radius $r_{n}$.

In our previous work we already considered RVEs with variable size. To this end we studied the distribution of the Voronoi cells area associated with corresponding inclusions. The Voronoi cell of an inclusion with center $\left(x_{1}^{c}, x_{2}^{c}\right)$ is the set of points which are closer to $\left(x_{1}^{c}, x_{2}^{c}\right)$ than to the center of any other inclusion.

Due to the small amount of real samples and lack of experimental data we generated virtual samples with completely randomly distributed inclusions and performed Voronoi analysis of these samples. The experimental distribution density function of the characteristic cell size $a(\omega)=\sqrt{A(\omega)} / 2$ was fitted with the log-normal rule.

The disadvantage of the scheme presented earlier is that Voronoi tessellation does not use any information about the inclusions' radii. On the one hand this fact strongly simplified the model. The area distribution was completely independent from the radii distribution. Both random variables $r(\omega)$ and $a(\omega)$ were uncorrelated, both distributions were fitted with the log-normal rule, thus simplifying a change of variables

$$
r_{n}(\omega)=\frac{r(\omega)}{a(\omega)}
$$

where $r_{n}(\omega)$ is log-normal too. On the other hand this model is very coarse. Due to the fact that we loose correlation between the inclusions' radii and the corresponding cell areas the area of some Voronoi cells can become smaller than the area of the corresponding inclusions. Moreover the Voronoi cell edges may intersect the inclusions boundaries (fig. 11 a). Thus in the present work we propose an improved model void of these disadvantages.

In the present study we use instead of the Voronoi diagram the so-called Apollonius diagram (additively weighted Voronoi diagram), its dual is the Apollonius graph also sometimes called the Delaunay graph of disks (fig. 11b). The computation of the 
Apollonius graph is a non-trivial problem due to the highly complex predicates and curvilinear edges of the Apollonius diagram. In the general case edges are hyperbolic curves. We used a specialized package of the Computational Geometry Algorithms Library (40) in order to compute the Apollonius diagrams.

Thus we generated 100000 inclusions with random radius according to the given experimental cumulative distribution function. We generated firstly the 100000 random numbers possessing Gaussian distribution using the standard random generator in Matlab. Then we replaced the samples laying outside the support of the truncated Gaussian distribution thus performing truncation. After that we compute the mapping of the truncated Gaussian distribution into the given experimental distribution (16). Using the obtained function we mapped the set of Gaussian random numbers into the set of random numbers with experimental distribution.

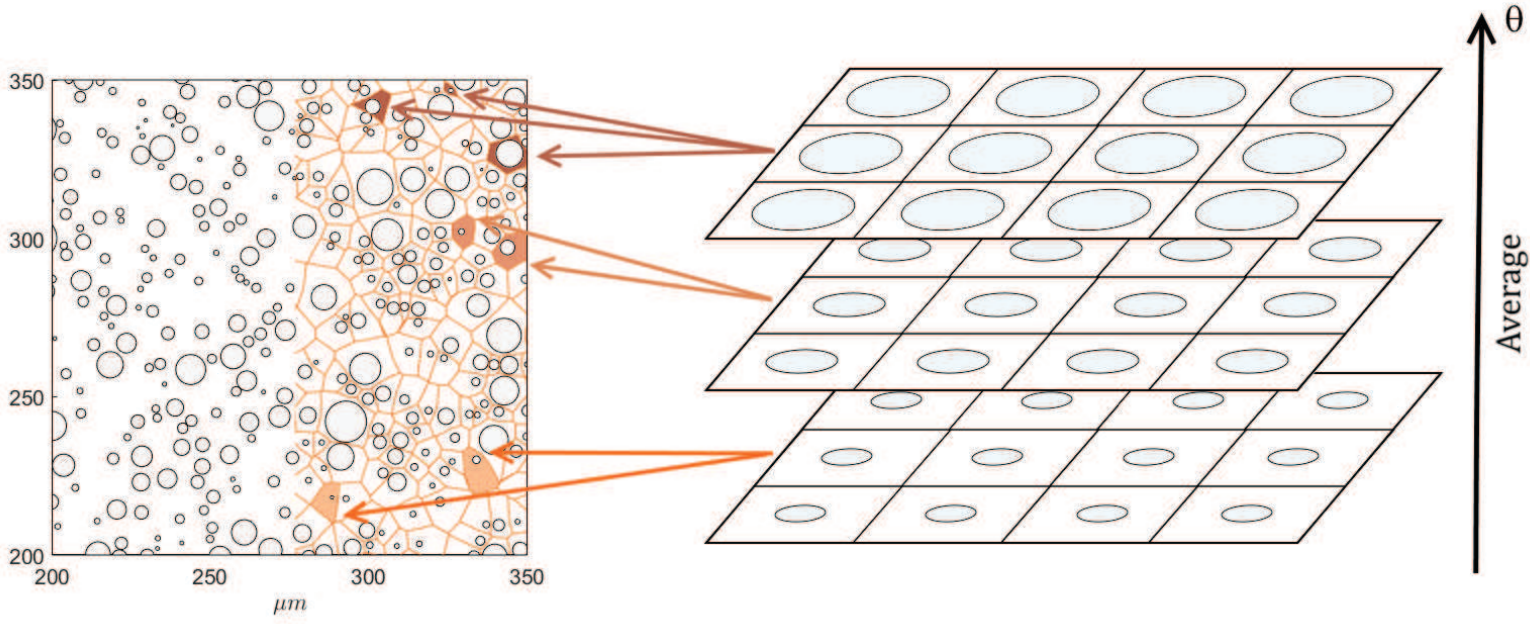

Figure 10: Virtual sample with randomly distributed particles (left) and the statistically similar stochastic periodic model (right). Ergodicity assumption: the average over ensembles is assumed equivalent to the average over the volume of the deterministic model with randomly distributed particles. Statistical similarity: probability density of the ratio between particle size and cell area, and the averaged volume fraction are the same in both models.

The size of the virtual material sample is chosen based on the volume fraction $v$ which is the relation of the total area of all inclusions to the area of the sample. Thus the area of the sample $A^{\text {sample }}$ is given as

$$
A^{\text {sample }}=v^{-1} \sum_{i=1}^{1 e 5} \pi r_{i}^{2} .
$$

Note that here we can specify the volume fraction in contrast to the model with fixed RVE size.

The generated inclusions were distributed inside the sample without overlapping. Periodicity was considered, thus the overlapping of the inclusions on opposite sides of the sample is also avoided. Figs. 10 and 11 demonstrate only a very small part of the obtained virtual random sample. 


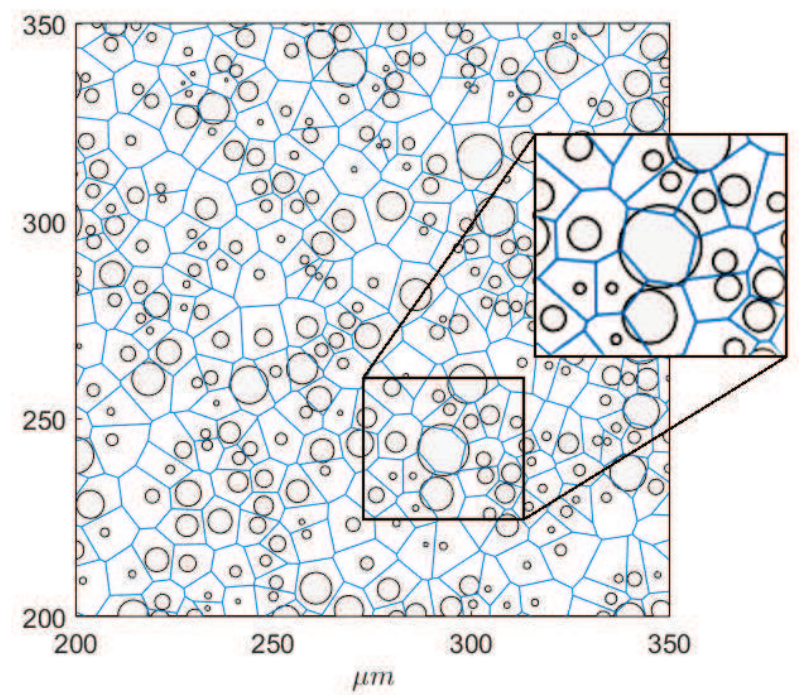

(a)

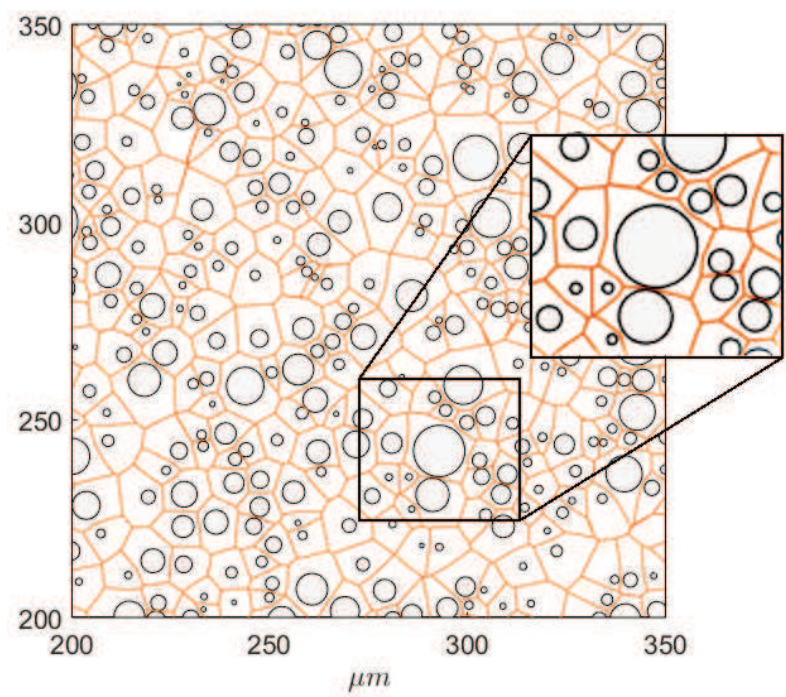

(b)

Figure 11: Part of the virtual sample with non-overlapping inclusions divided into divided into Voronoi cells (a) and Apollonius cells (b). Note Voronoi edges intersecting inclusions' boundaries.

For the generated virtual sample of material we calculate the Apollonius diagram with periodic boundary conditions. A part of the Apollonius diagram is depicted in fig. 11b. The areas $A_{i}^{\text {cell }}$ of the non-convex curvilinear Apollonius cells are computed numerically. The characteristic size of the cell $a$ is evaluated as $a_{i}=\sqrt{A_{i}^{\text {cell }}} / 2$.

Due to the fact that the variable size of the RVE is inconvenient for the evaluation of the homogenized properties we introduce the reduced radius $r_{n i}=r_{i} / a_{i}$ similar to the model proposed in (65). Thereby we keep the size of the RVE fixed and compress two random parameters into one thus strongly reducing the computational effort.

The weight of every single realization of $r_{n}$ is the total area of all cells possessing corresponding $r / a$-ratio. Fig. 12 demonstrates the statistical area distribution plotted versus the reduced radius $r_{n}$. The dashed area in the right part of the figure denotes physically impossible values of $r_{n}$. If $r_{n} \geq \sqrt{\frac{\pi}{4}}$ the area of the inclusion is bigger than the area of the Apollonius cell, thus impossible for the non-overlapping inclusions. However this is the case for Voronoi tessellation. Two vertical blue lines $r_{n}=0.9$ and $r_{n}=0.09$ are limits covering $99.92 \%$ of entire sample area. Cells lying outside these limits are depicted by blue color. Only the cells inside these limits are used for the evaluation of the statistical cdf.

The interval between $r_{n}=0.9$ and $r_{n}=0.09$ is divided into 200 subintervals $d r_{n i}$. The total area of all cells in the interval $d r_{n i}$ (fig. 12) divided by the area of the material sample represents the probability mass function over this interval. The cumulative sum of all mass functions approximate the cumulative distribution function of $r_{n}(\omega)$. Note that here we do not use the statistical $r_{n i}$ but the probabilistic $r_{n}(\omega)$. The experimental cdf of the $r_{n}(\omega)$ is depicted in fig.13,

The obtained cdf is much closer to the truncated Gaussian distribution than to the log-normal distribution. However the fitting with the truncated Gaussian distribution fails. The purple dashed line in fig. 13 demonstrates the probability density function of the experimental distribution obtained by simple differentiation of the experimental cdf. The experimental pdf is asymmetric 


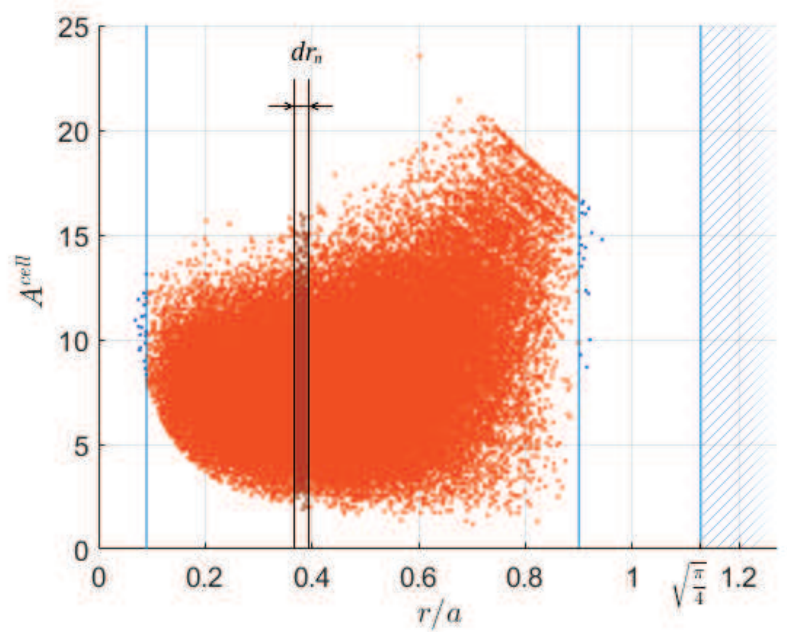

Figure 12: Area of the Apollonius cells $A^{\text {cell }}$ plotted versus $r_{n}=r / a$. The dashed area in the right part of the picture denotes physically impossible values of $r_{n}$.

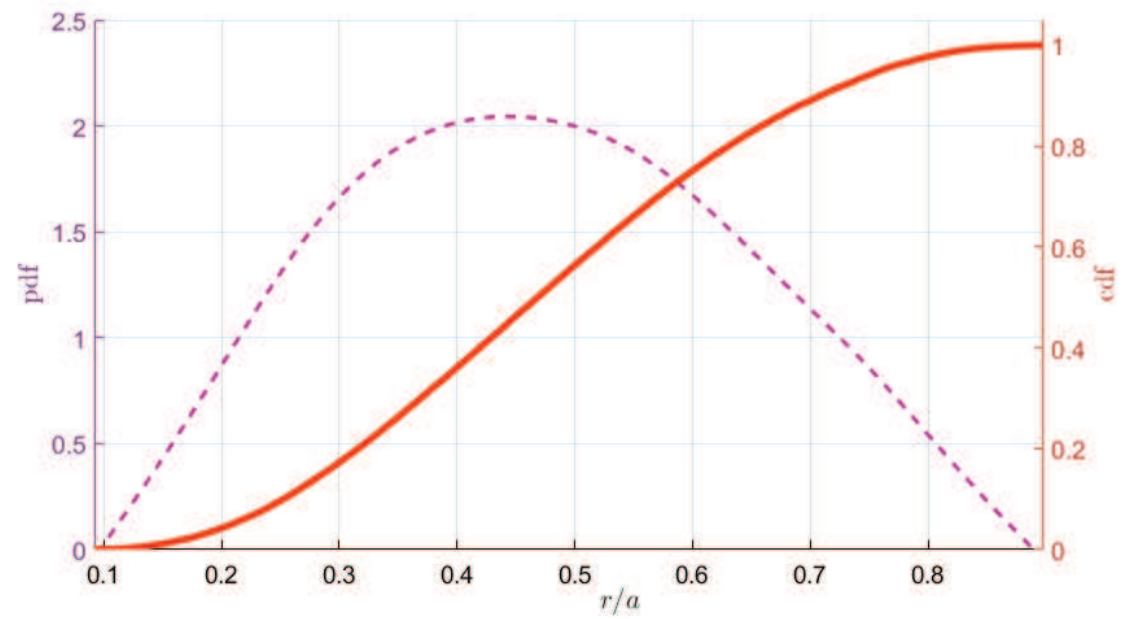

Figure 13: Experimental cumulative distribution function and probability density function of the reduced radius $r_{n}(\omega)$.

and exhibits no tails typical for Gaussian distribution. An attempt to fit the experimental cdf with Gaussian distribution (fig. 14) results in negative minimum radius value and an extremely wide range of radii variation. Thus the reduced radius $r_{n}(\omega)$ should be represented as nonlinear mapping of the truncated Gaussian basis RV.

The mapping curve $r_{n}(\theta(\omega)): \theta(\omega) \rightarrow \mathbb{R}$ is computed from expression (16). The obtained function exhibits a shape very similar to a cubic polynomial (fig. 15). This curve represents an exact expression for the random radius in terms of the basic random variable. However, for the application within the SFEM the parametrized function is preferred, thus we should fit 


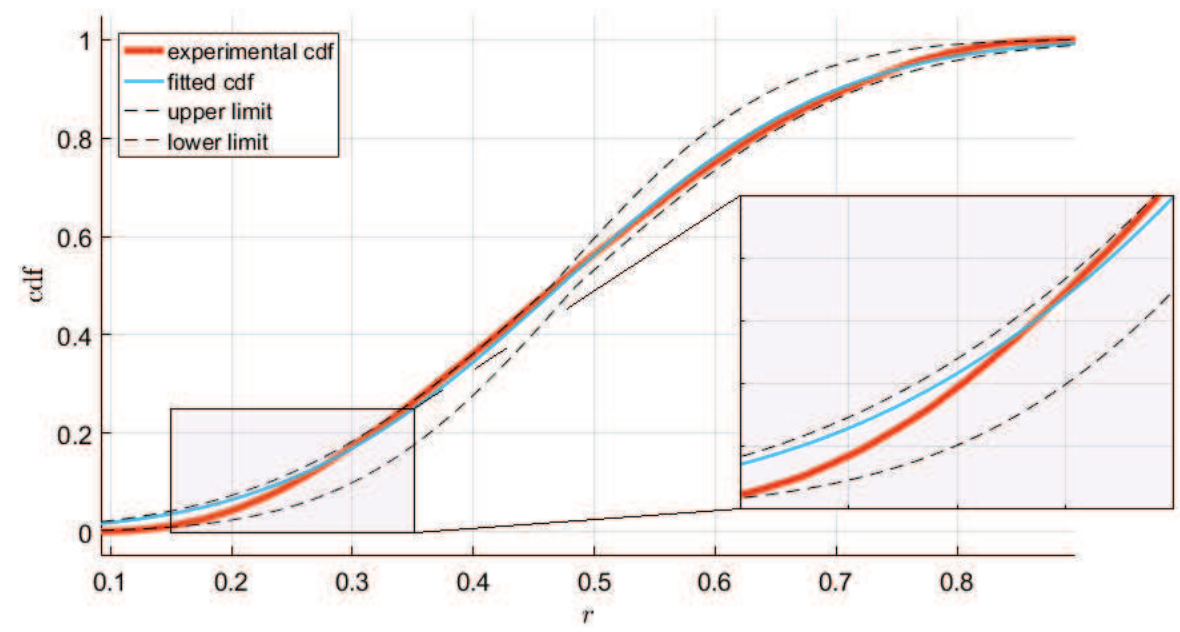

Figure 14: Fitting of the experimental curve $r_{n}(\omega)$ with truncated Gaussian variable. Obtained maximum and minimum radii contradict with physical limitations.

the tabulated curve $r_{n}(\theta(\omega))$ using some analytical model. To this end we introduce a new random variable which is a cubic polynomial of the truncated Gaussian variable $\theta(\omega)$.

$$
\begin{aligned}
& p_{n}(\omega)=\sum_{i=1}^{4} a_{i} h_{i}(\theta(\omega)), \\
& h_{1}(\theta(\omega))=[1+2 \theta(\omega)][1-\theta(\omega)]^{2}, \\
& h_{2}(\theta(\omega))=\theta(\omega)[1-\theta(\omega)]^{2}, \\
& h_{3}(\theta(\omega))=\theta(\omega)^{2}[3-2 \theta(\omega)], \\
& h_{4}(\theta(\omega))=\theta(\omega)^{2}[\theta(\omega)-1],
\end{aligned}
$$

where $h_{i}$ are cubic Hermite splines.

This model includes 4 parameters $a_{i}$. The convenience of the Hermite representation is that two parameters, namely $a_{1}$ and $a_{3}$ are immediately evaluated from the tabulated data - they correspond to the function values at the ends of the interval. Thereby only parameters $a_{2}$ and $a_{4}$ should be fitted from the tabulated data. The necessary requirement

$$
\frac{\mathrm{d} p_{n}}{\mathrm{~d} \theta}>0
$$

is satisfied for the here considered curve if $a_{2}>0$ and $a_{4}>0$, also due to the convenient form of the Hermite representation. The fitting procedure is the same as that used to fit the experimental cdf. 
Fig. 15 demonstrates the original curve $r_{n}(\theta(\omega))$, the fitted Hermite spline, and the upper and lower bounds. Note the very good agreement between the original curve and the cubic polynomial. Fig. 16 demonstrates the original experimental cdf, the cdf of the cubic RV, and the corresponding bounds.

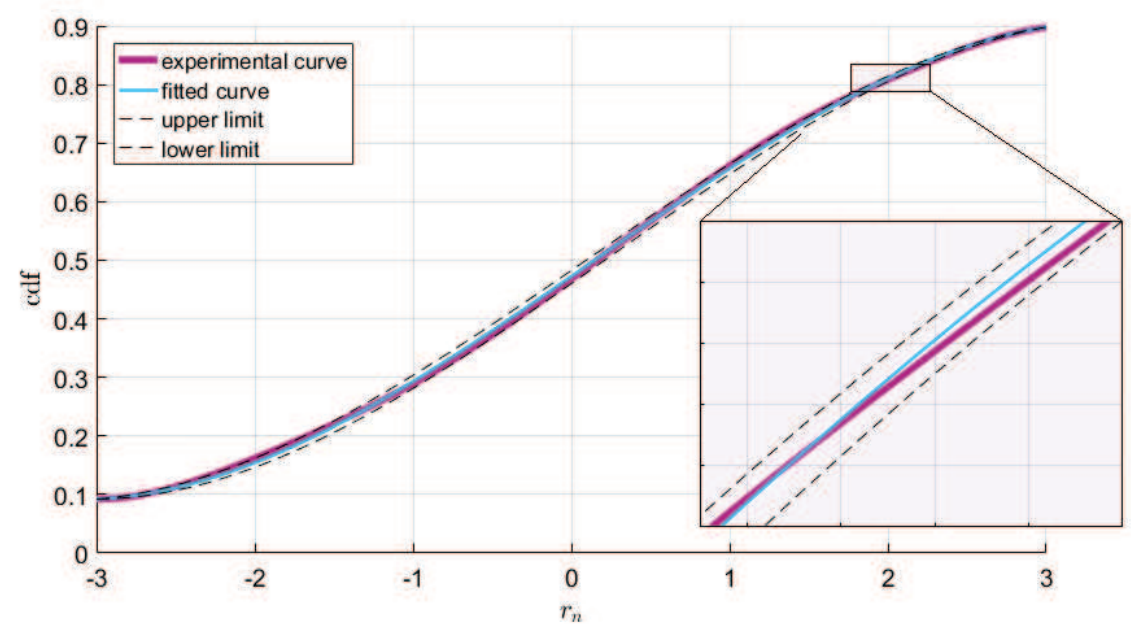

Figure 15: Fitting of the experimental curve $r_{n}(\theta(\omega))$ with the cubic polynomial of the truncated Gaussian variable $p_{n}(\theta(\omega))$.

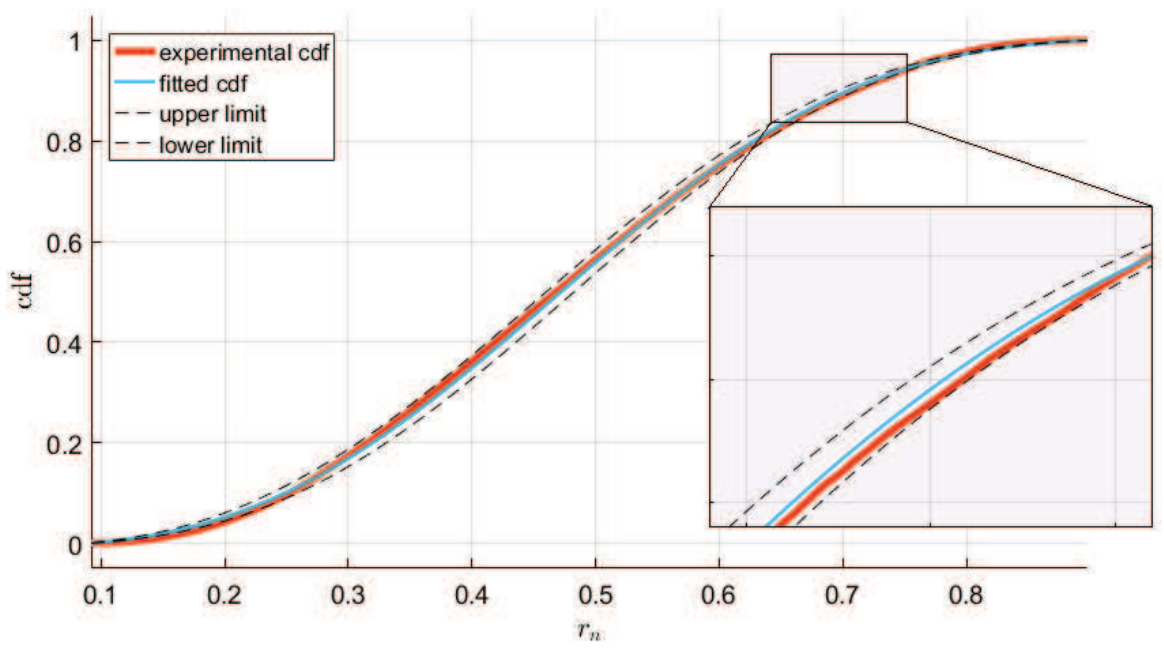

Figure 16: Experimental cumulative distribution function of the reduced radius $r_{n}(\omega)$ and cdf of the polynomial RV $p_{n}(\theta(\omega))$.

Based on the results of curve fitting we design two triangular fuzzy numbers: $\tilde{a}_{2}$ with modal value $\bar{a}_{2}=0.0729$ defined in the range $[0.0108,0.1340]$ and $\tilde{a}_{4}$ with modal value $\bar{a}_{4}=0.2542$ defined in the range $[0.2328,0.2892]$. Two crisp model parameters are $a_{1}=0.0920$ and $a_{3}=0.8980$.

Thereby the inclusions radius in (19) is described as cubic random variable with two fixed and two fuzzy parameters thus requiring the application of the fuzzy-stochastic FEM framework. 


\section{Simulation results for the RVE with fixed cell size}

The most accurate evaluation of the fuzzy system output for the general nonlinear non-monotonic dependence is obtained by using the optimization method. Due to the large number of samples required, this approach is costly. Thus the general transformation method (29, 30) is often applied in order to obtain an approximate response surface. However in some cases the number of samples required may be strongly reduced. E.g., in the case when the output function is monotonic only the corner points of each interval should be considered thus yielding the well-known full factorial design pattern (vertex method).

Due to the fact that we don't know a priori whether the output is monotonic (however we expect this), we can not use the vertex method immediately. Thus we perform first a relative simple simulation using the general transformation method in order to prove that the output is monotonic. After that, if the output demonstrates monotonic response, the vertex method is applied to the original and all further problems.

The transformation method is highly efficient in the case of symmetric triangular fuzzy numbers, otherwise the number of samples required increases. Thus we perform firstly a simulation with symmetric fuzzy numbers. Thereby we extend the support of the fuzzy numbers to the left and to the top (compare supports in figs. 17]and 18). Fig. 17illustrates sampling with the general transformation method for the case of five $\alpha$-cuts. Black lines denote the edges of the membership function, which represents for the case of two triangular fuzzy numbers a pyramid in the space $\left(m_{r}, \sigma_{r}, \mu_{m \sigma}\right)$. The sampling of two asymmetric fuzzy numbers with the vertex method is depicted in fig. 18 Empty circles denote optional samples. In the case of a linear output function or in the case when the reconstruction of the fuzzy output is not required, these additional samples are not necessary.

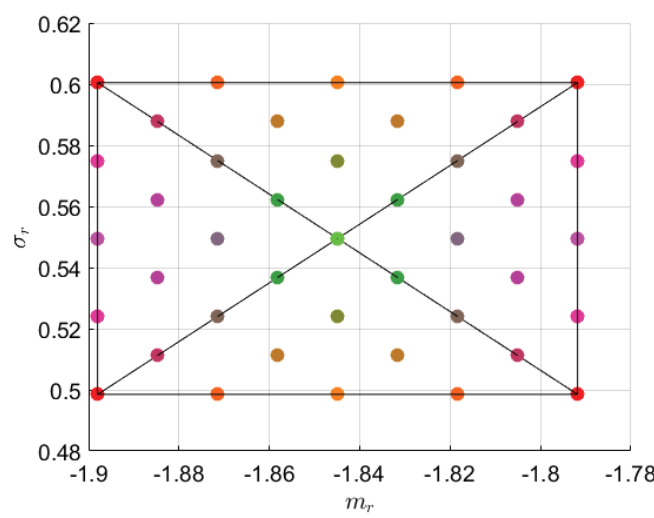

Figure 17: Sampling for the case of the general transformation method applied to the pair of symmetric fuzzy numbers.

The aim of this simulation is to study the influence of the fuzzy distribution parameters, obtained from the experiment, on the homogenized stress values. Thus the fuzzy output represents here the homogenized stress plotted versus the basic random variable. Every fuzzy sample is resolved using the isoparametric SL-FEM with quadratic finite elements, whose accuracy 


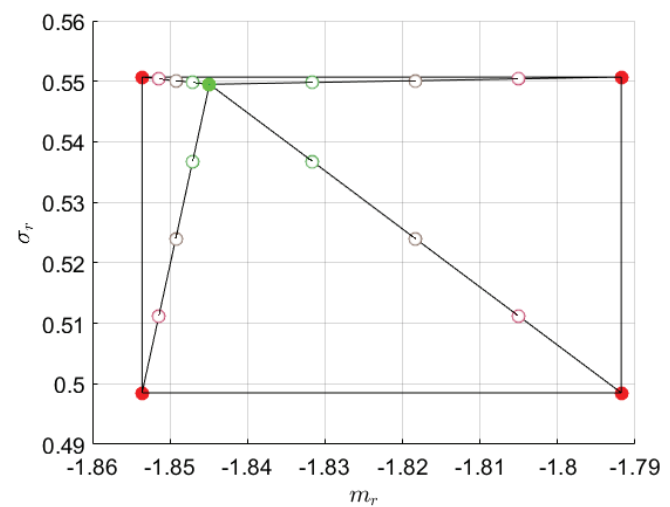

Figure 18: Sampling of two fuzzy numbers using the vertex method. Empty circles denote samples, which are not necessary, if, e.g., the output is a linear function of the model parameters.

and convergence for the considered problem was studied in (65). For the homogenized stress quantities the SL-FEM exhibits exponential convergence already starting from the four element layers in the stochastic dimension.

In the present study the physical-stochastic product domain $\mathcal{V}$ is discretized using 16 element layers in the stochastic dimension thus yielding very high accuracy for the homogenized stress quantities. The finite element mesh is generated from the same pattern for every sample, thus the number of elements stays unchanged. The mesh generated for the modal values of fuzzy parameters is depicted in fig. 19. Lilac is used to denote inclusion, orange corresponds to the elastomer matrix.

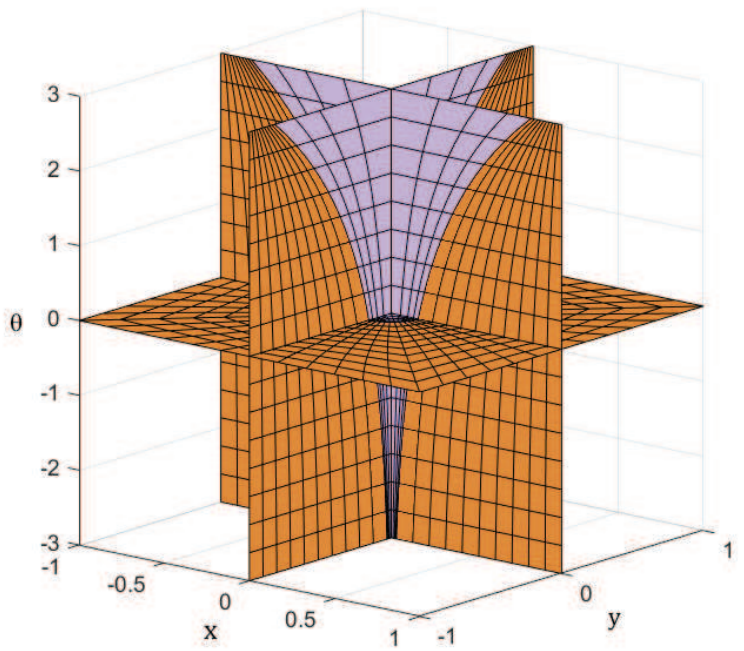

Figure 19: Finite element discretization of the physical-stochastic domain $\mathcal{V}$. Model with fixed RVE size. Lilac denotes inclusion, orange corresponds to the elastomer matrix.

The fuzzy response curves for the case of symmetric fuzzy numbers are presented in fig. 20 for the entire support of the basic RV $\theta(\omega) \in[-3,3]$ and in fig. 21 for the subinterval of the most probable values $\theta(\omega) \in[-1,1]$. Different curve colors correspond to the samples in fig. 17. The analysis of the presented curves demonstrates that the output function is monotonic 
with respect to both fuzzy parameters $\tilde{m}_{r}$ and $\tilde{\sigma}_{r}$ however their influence on the system behavior is different. The variation of $m_{r}$ moves the entire curve up and down, while the variation of $\sigma_{r}$ "rotates" the curve around the center point $\theta(\omega)=0$. Thereby maximum and minimum values of the fuzzy output are reached in the corner points of the interval. Fig. 22. $\mathrm{represents} \mathrm{the} \mathrm{stress}$ curves corresponding to the min and max values of the fuzzy output.

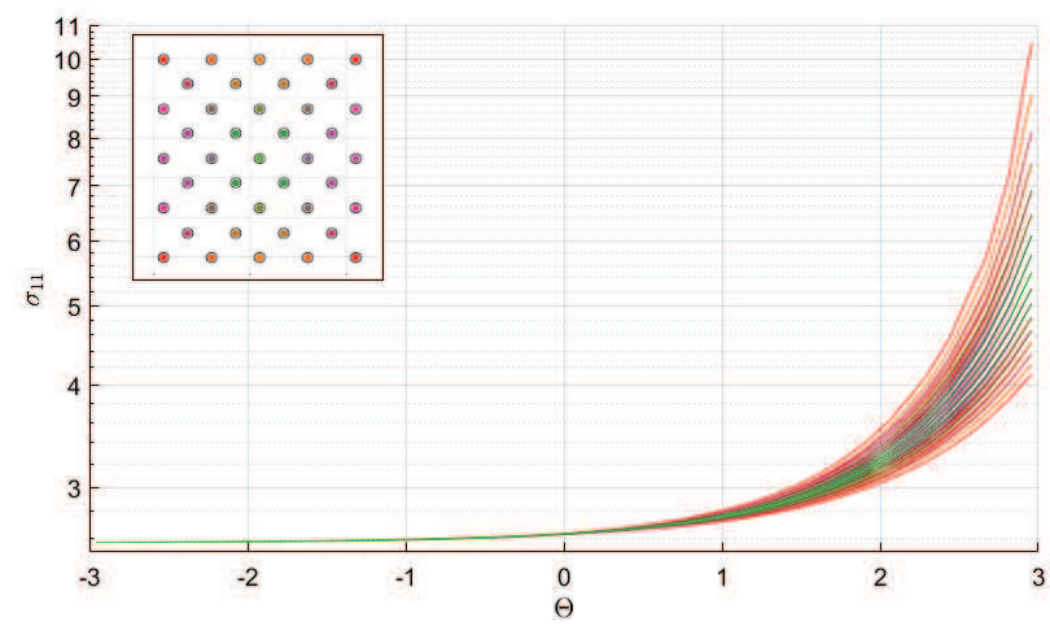

Figure 20: Stochastic homogenized stress curves plotted versus basic RV $\theta(\omega) \in[-3,3]$. Different curve colors correspond to the samples in fig. 17, Model with fixed RVE size.

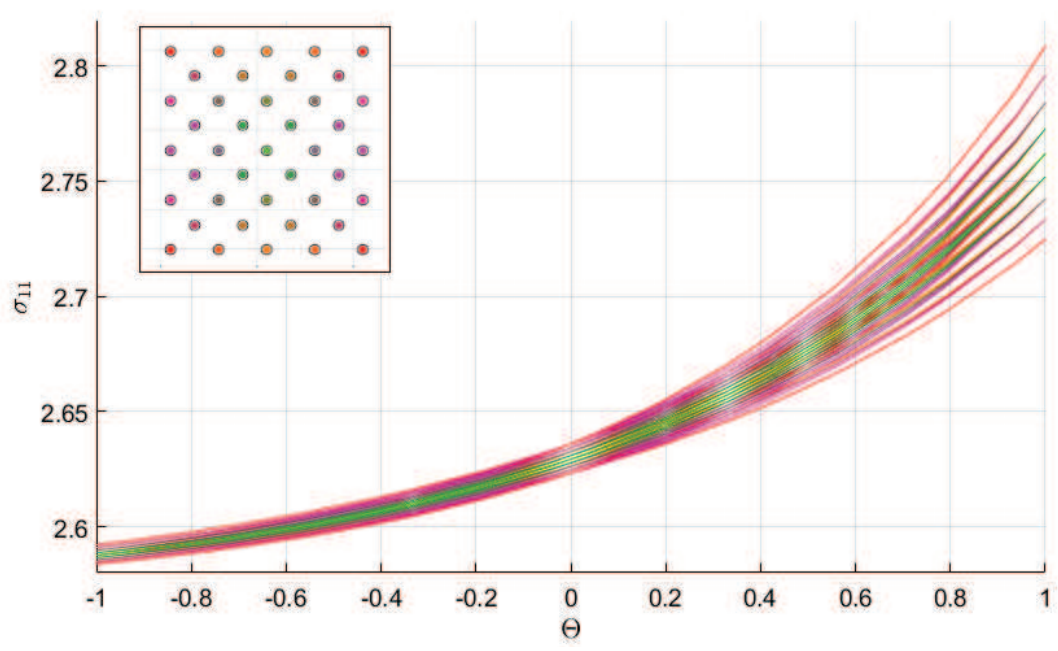

Figure 21: Stochastic homogenized stress curves plotted versus basic RV $\theta(\omega) \in[-1,1]$. Different curve colors correspond to the samples in fig.17. Model with fixed RVE size.

Fig. 23 demonstrates the homogenized stress mean value and STD for different samples. Both the mean value and the STD reach their maximum and minimum values in the corner points. Therefore all three quantities of interest (stochastic 
homogenized stress, homogenized stress mean value, and homogenized stress STD) demonstrate monotonic behavior thus allowing the use of the vertex method for further simulations.

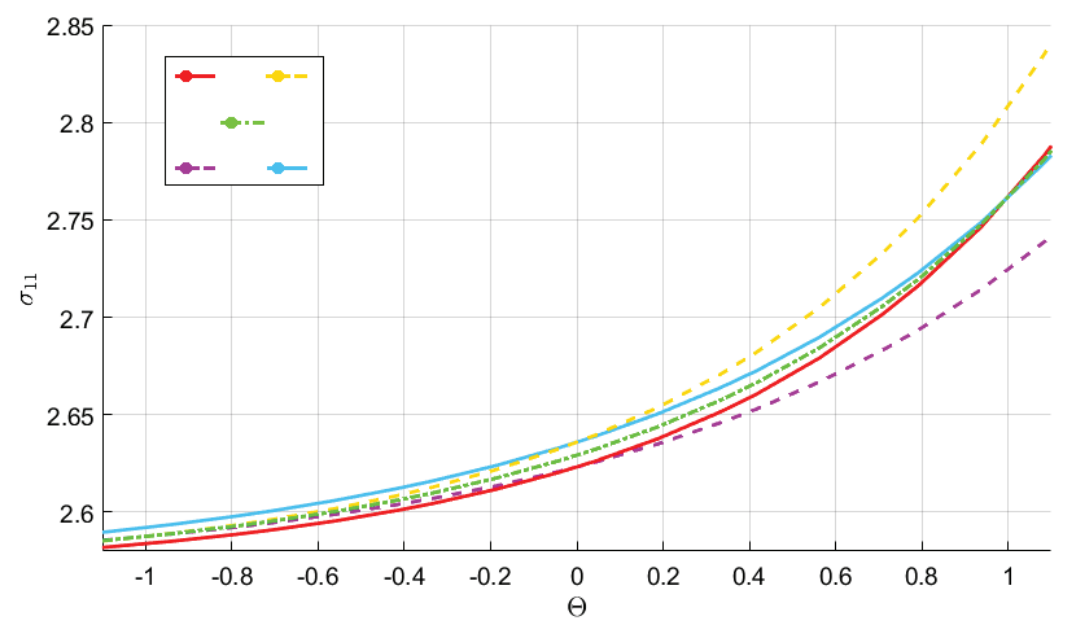

Figure 22: Stress curves corresponding to the min and max values of the fuzzy output. Model with fixed RVE size.
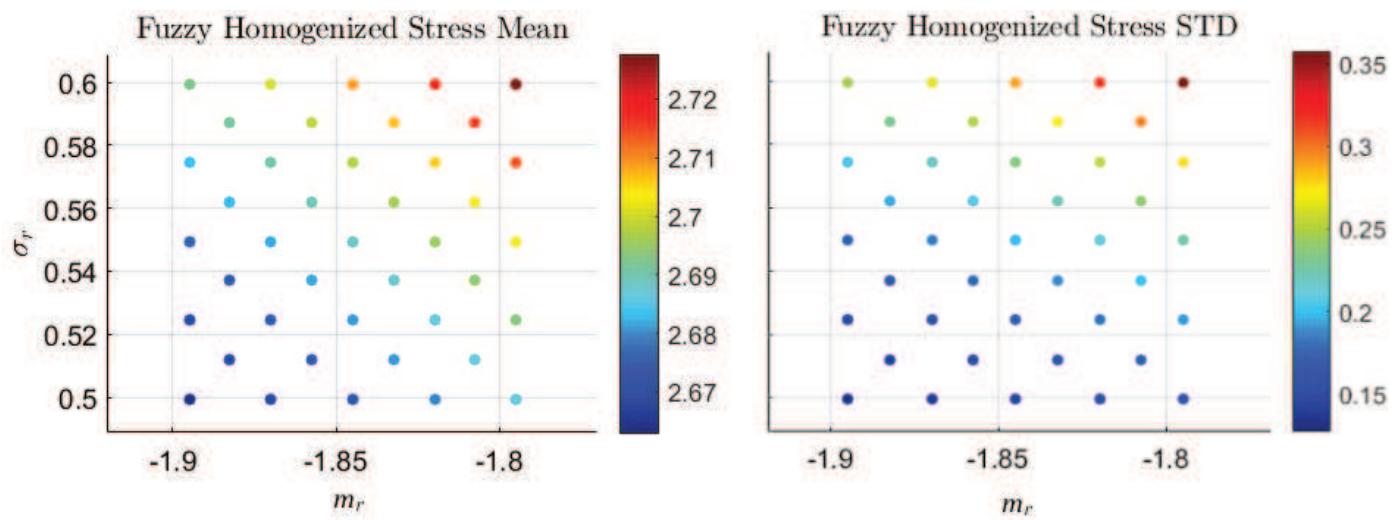

Figure 23: Homogenized stress mean value and STD plotted in the sample space $\left(\tilde{m}_{r}, \tilde{\sigma}_{r}\right)$. Model with fixed RVE size.

Fig. 24 demonstrate the stochastic homogenized stress curves obtained using the vertex method (fig. 18). The curve's shape is close to exponential. This is expected due to the log-normal rule of the radii distribution. Note the strong influence of the parameter variation on the homogenized stresses. The maximum homogenized stress value increases, e.g., by $50 \%$, However the variation of the distribution parameters was around 5-10\%.

Note also that the homogenized stress STD (fig.23) differs 2.5 times for different samples. Thus the model is highly sensitive to the input variations, therefore the accuracy of the model predictions depend strongly on the accuracy of the experimental data and statistical estimations. 


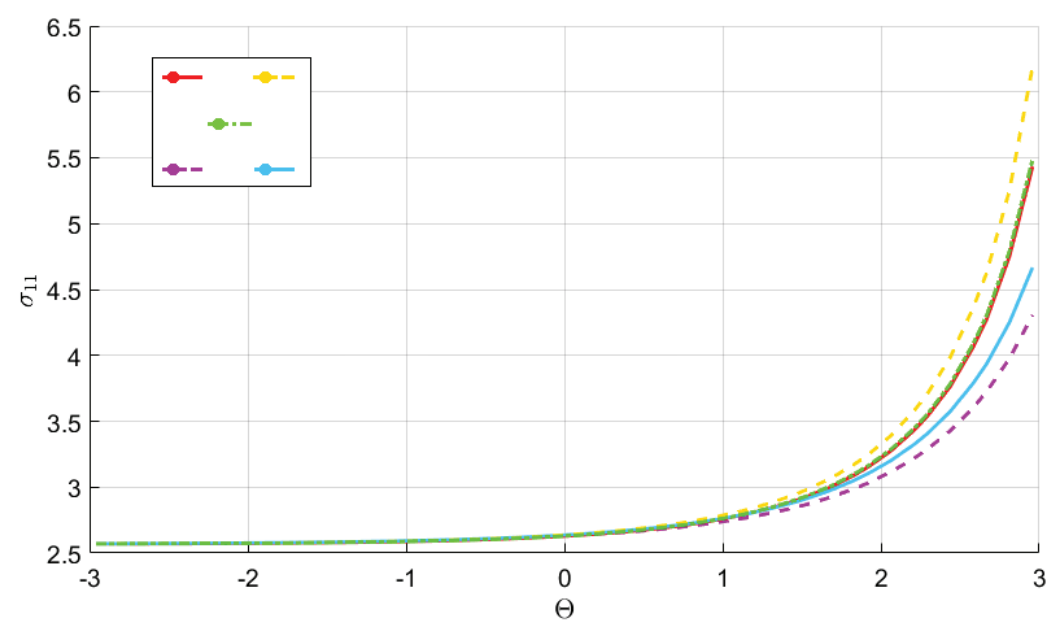

Figure 24: Stress curves corresponding to the min and max values of the fuzzy output. Solution for the non-symmetric fuzzy number was obtained using the vertex method. Model with fixed RVE size.

\section{Simulation results for the RVE with variable cell size}

For the here considered problem it is difficult to define a priori whether the system output is monotonic with respect to the fuzzy parameter variations. Thus the vertex method cannot be applied. Due to the fact that both fuzzy parameters are almost symmetric, the general transformation method is preferred. The sampling of the parameter space is depicted in fig. 25.

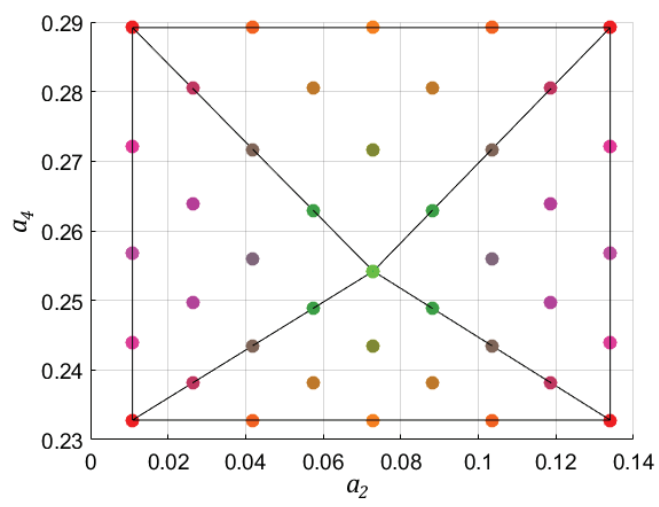

Figure 25: Sampling strategy for the model involving a cubic random variable with two triangular fuzzy parameters.

The simulations presented in this section were performed using 12 element layers in the stochastic dimensions due to the smaller radius variation and also smaller rate of radius increase. The mesh generated for the modal values of fuzzy parameters is depicted in fig. 26. Lilac is used to denote inclusion, orange corresponds to the elastomer matrix.

The obtained stochastic homogenized stress curves (fig. 27) demonstrate higher average stress values, which is expected due to the higher volume fraction in this model ( $20 \%$ versus $3 \%$ in the model with fixed RVE size). The obtained curves demonstrate also a smaller spread compared to the log-normal distribution, which may be considered as a very important computational 


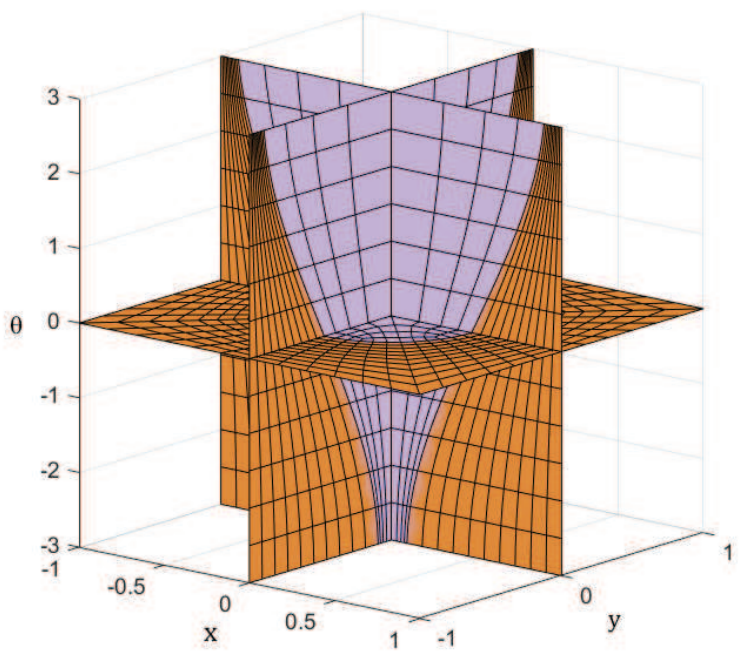

Figure 26: Finite element discretization of the physical-stochastic domain $\mathcal{V}$. Model with variable RVE size. Lilac denotes inclusion, orange corresponds to the elastomer matrix.

advantage of the model with variable RVE size. In order to analyze the boundaries of the stress curves, we plot also the stresses subtracting the modal values $\sigma_{11}-\bar{\sigma}_{11}=\sigma_{11}\left(a_{2}, a_{4}\right)-\sigma_{11}\left(\bar{a}_{2}, \bar{a}_{4}\right)$ (fig. 28).

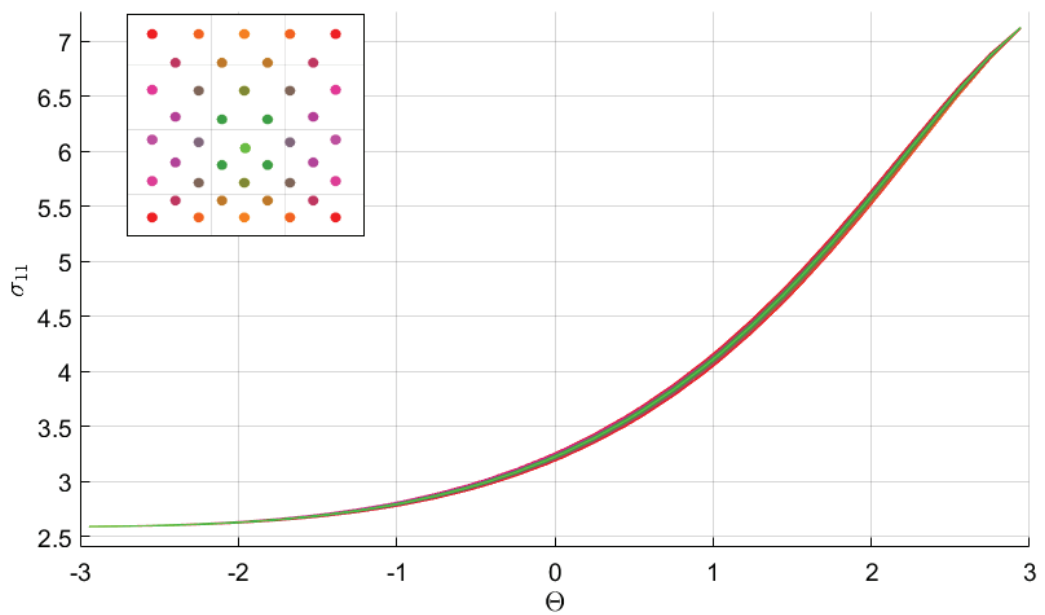

Figure 27: Stochastic homogenized stress curves plotted versus basis RV $\theta(\omega) \in[-3,3]$. Different curve colors correspond to the samples in fig. 25. Model with variable RVE size.

The max and min stress values for every $\alpha$-cut are presented in fig. 29 Note that the max and min curves coincide with the samples located in the vertices of the $\alpha$-cut, thus demonstrating monotonic dependence of the homogenized stresses on the distribution parameters $\tilde{a}_{2}$ and $\tilde{a}_{4}$.

Homogenized stress mean value and STD are depicted in fig. 30. The model response demonstrates the monotonic behavior with respect to parameters $\tilde{a}_{2}$ and $\tilde{a}_{4}$. Note also the small variation of the stress STD, which states that the model with variable RVE size is less sensitive to the variation of the input parameters. Interesting is the fact that the stress mean value increased 


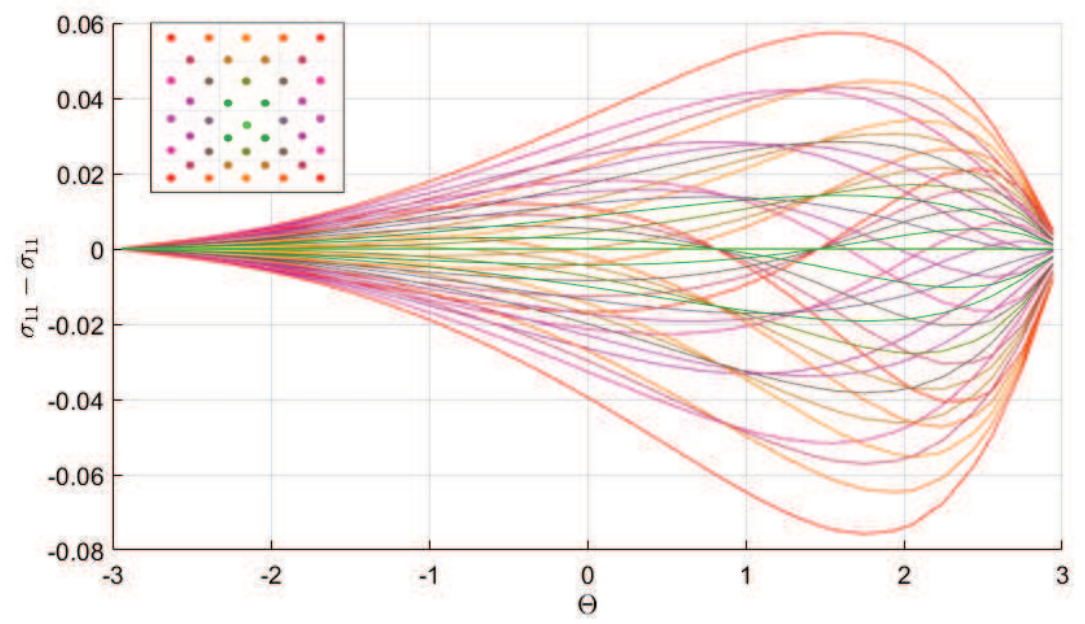

Figure 28: Deviation of the homogenized stresses from the modal values $\sigma_{11}-\bar{\sigma}_{11}=\sigma_{11}\left(a_{2}, a_{4}\right)-\sigma_{11}\left(\bar{a}_{2}, \bar{a}_{4}\right)$ plotted versus the basic random variable $\theta(\omega)$. Model with variable RVE size.

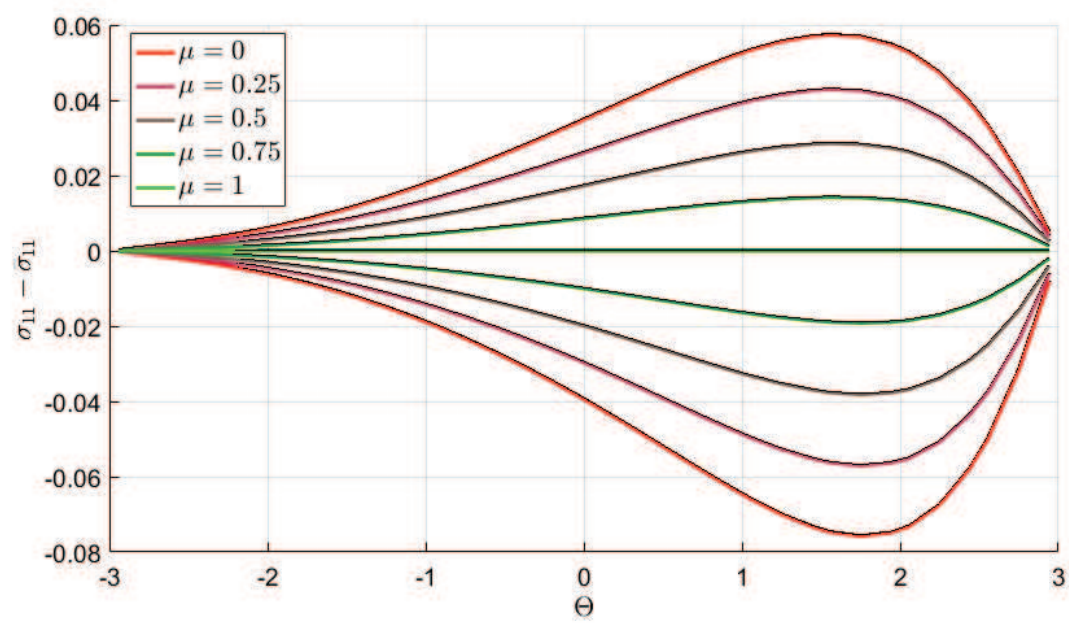

Figure 29: Stress boundaries for each $\alpha$-cut plotted in form of deviation from the modal value $\sup \left\{\sigma_{11}^{\alpha}\right\}-\bar{\sigma}_{11}$ and $\inf \left\{\sigma_{11}^{\alpha}\right\}-$ $\bar{\sigma}_{11}$. Model with variable RVE size.

approximately 1.25 times compared to the model with fixed size of the RVE, however the volume fraction increased from 3\% to $20 \%$.

\section{Summary and conclusions}

In the present work we established a combined fuzzy-stochastic FEM and implemented the proposed technique into computational homogenization. Based on the analysis of experimental evidence we specified two different sources of uncertainty in the materials' microstructure requiring different solution strategies. 

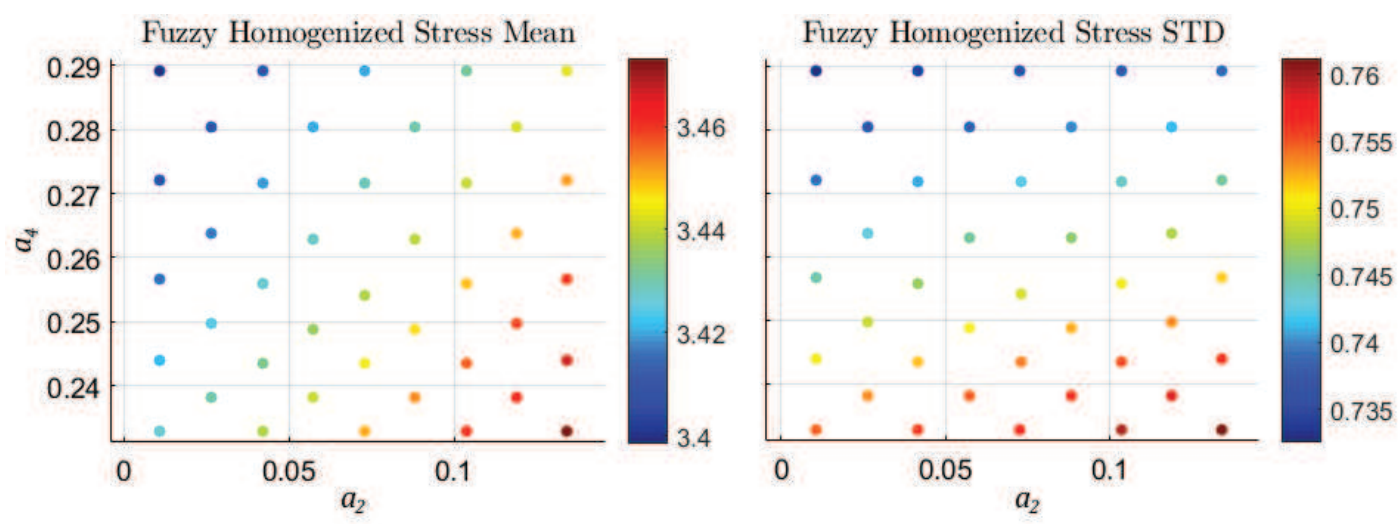

Figure 30: Homogenized stress mean value and STD plotted in the sample space $\left(\tilde{a}_{2}, \tilde{a}_{4}\right)$. Model with variable RVE size.

The randomness in the geometry of the microstructure resulted from the variability of inclusions' radii and random inclusions' distribution. This aleatoric uncertainty requires probabilistic descriptions. The distribution parameters were estimated statistically directly from the experimental data.

The particle distribution was assumed to be completely random, thus the probabilistic description in terms of probability density function, random variables, etc is highly complicated. An attempt to describe the inclusions' positions in terms of RVs results in a large and highly complicated system of dependent RVs completely useless for engineering applications. Thus we proposed some alternative description of the inclusions' position distribution based on the concept of Voronoi cells — the region of the matrix material which is closer to the inclusion associated with it than to any other inclusion. If the inclusions radius is considered, the Apollonius diagram is used. Based on the analysis of virtual material samples we estimated statistically the relation between the inclusion's radius and the corresponding Apollonius cell area distribution and used this information to design the stochastic RVE with variable size.

In this work we analyzed two models — with fixed RVE size and with variable RVE size. For both models of the stochastic RVE we demonstrated the sources of epistemic uncertainty. Due to the idealized and simplified character of parametric probability distribution functions the fitting of the experimental curves results in the distribution parameters' values lying within some interval of confidence. The natural way to describe epistemic uncertainty is fuzzy arithmetic operating rather with possibilities than with probabilities.

Thus the choice of fuzzy-stochastic solution strategies is motivated empirically.

Fuzzy output was presented by scattered response surface plots (figs. 23 and 30) and boundary curves (figs. 24 and 29) demonstrating the output's variation for every considered $\alpha$-cut. Thus the fuzzy response curves allow to estimate the interval of confidence for quantities of interest if the input is epistemic. They demonstrate also how the interval of confidence could change if more effort would be invested into the experiment in order to reduce the epistemic uncertainty (chapter 3.1) and also how the consideration of different fits (obtained using different fitting algorithms or weights) affects the output quantities. Information provided allowed complete analysis of the effect of epistemic uncertainty on the stochastic output. 
Based on the performed simulations we conclude that the influence of the input's fuzziness on the simulation results is large. The maximum homogenized stresses may change by $50 \%$ with only $5 \%$ input parameters' variation. For the model with fixed RVE size the homogenized stress STD may change more than two times. The model with variable RVE size is more stable and demonstrates a stress change comparable to the variation of input parameters.

For both RVE models studied the system's response demonstrated monotonic dependence on the input parameters thus allowing later application of the vertex method. However this fact is related only to the proposed models and cannot be generalized to other RVEs.

From the comparison of the RVE with fixed size and the RVE with variable size we conclude that the RVE with variable size is less sensitive to the variation of fuzzy parameters, produces stress curves with smaller spread, gives control over the inclusion's volume fraction, captures more statistical and experimental information related to the microstructure, however the design of the stochastic RVE based on the experimental data becomes more sophisticated and requires additional calculations.

Both methods, the stochastic FEM technique and the general transformation method for fuzzy numbers, are computationally expensive thus motivating the future incorporation of reduced order techniques into the SFEM framework

Also the development of advanced sampling techniques for fuzzy numbers may be used in order to accelerate the solution of the combined fuzzy-stochastic problem.

\section{Acknowledgments}

The support of this work by the ERC Advanced Grant MOCOPOLY and Deutsche Forschungs-Gemeinschaft (DFG) through the Priority Program SPP1886 and through the DFG Grant FOR 2271 is gratefully acknowledged. The authors also thank Bastian Walter for preparation of elastomer samples and providing corresponding SEM images.

\section{References}

1. Adhikari S. A reduced spectral function approach for the stochastic finite element analysis. Computer Methods in Applied Mechanics and Engineering. 2011;200(21-22):1804-1821.

2. Alsayednoor J., Harrison P., Guo Z. Large strain compressive response of 2-d periodic representative volume element for random foam microstructures. Mechanics of Materials. 2013;66(0):7 -20.

3. Andrianov Igor V., Danishevs'kyy Vladyslav V., Kholod Elena G. Homogenization of viscoelastic composites with fibres of diamond-shaped cross-section. Acta Mechanica. 2012;223(5):1093-1100.

4. Andrianov Igor V., Danishevs'kyy Vladyslav V., Weichert Dieter. Asymptotic determination of effective elastic properties of composite materials with fibrous square-shaped inclusions. European Journal of Mechanics - A/Solids. 2002;21(6):1019 -1036.

5. Andrianov IgorV., Danishevs'kyy Vladyslav V., Weichert Dieter. Simple estimation on effective transport properties of a random composite material with cylindrical fibres. Zeitschrift fuer angewandte Mathematik und Physik. 2008;59(5):889-903. 
6. Arregui-Mena José David, Margetts Lee, Mummery Paul M. Practical application of the stochastic finite element method. Archives of Computational Methods in Engineering. 2016Mar;23(1):171-190.

7. Babuska Ivo, Chatzipantelidis Panagiotis. On solving elliptic stochastic partial differential equations. Computer Methods in Applied Mechanics and Engineering. 2002;191(37-38):4093-4122.

8. Babuska Ivo, Motamed Mohammad. A fuzzy-stochastic multiscale model for fiber composites: A one-dimensional study. Computer Methods in Applied Mechanics and Engineering. 2016;302:109 -130.

9. Babuska Ivo, Nobile Fabio, Tempone Raul. A stochastic collocation method for elliptic partial differential equations with random input data. SIAM Journal on Numerical Analysis. 2007;45(3):1005-1034.

10. Babuska Ivo, Tempone Raul, Zouraris Georgios E. Galerkin finite element approximations of stochastic elliptic partial differential equations. SIAM Journal on Numerical Analysis. 2004;42(2):800-825.

11. Babuska Ivo, Tempone Raul, Zouraris Georgios E. Solving elliptic boundary value problems with uncertain coefficients by the finite element method: the stochastic formulation. Computer Methods in Applied Mechanics and Engineering. 2005;194(12-16):1251 -1294 Special Issue on Computational Methods in Stochastic Mechanics and Reliability Analysis.

12. Bris Claude Le, Legoll Frederic. Examples of computational approaches for elliptic, possibly multiscale pdes with random inputs. Journal of Computational Physics. 2017;328(Supplement C):455 -473.

13. Castaneda P. Ponte, Galipeau E. Homogenization-based constitutive models for magnetorheological elastomers at finite strain. Journal of the Mechanics and Physics of Solids. 2011;59(2):194 -215.

14. Chatzigeorgiou George, Javili Ali, Steinmann Paul. Unified magnetomechanical homogenization framework with application to magnetorheological elastomers. Mathematics and Mechanics of Solids. 2013;2012:193-211.

15. Chevreuil M., Nouy A., Safatly E. A multiscale method with patch for the solution of stochastic partial differential equations with localized uncertainties. Computer Methods in Applied Mechanics and Engineering. 2013;255:255 -274.

16. Cottereau R., Clouteau D., Ben Dhia H. Localized modeling of uncertainty in the arlequin framework,In Belyaev Alexander K., Langley Robin S. (eds.) 2011 (pp. 457-468)., Iutam symposium on the vibration analysis of structures with uncertainties: Springer Netherlands.

17. Cottereau Regis. A stochastic-deterministic coupling method for multiscale problems. application to numerical homogenization of random materials. Procedia IUTAM. 2013;6(0):35 -43 IUTAM Symposium on Multiscale Problems in Stochastic Mechanics.

18. Deb Manas K., Babuska Ivo M., Oden J.Tinsley. Solution of stochastic partial differential equations using galerkin finite element techniques. Computer Methods in Applied Mechanics and Engineering. 2001;190(48):6359 -6372.

19. Dimas Leon S., Giesa Tristan, Buehler Markus J. Coupled continuum and discrete analysis of random heterogeneous materials: Elasticity and fracture. Journal of the Mechanics and Physics of Solids. 2014;63(0):481 -490.

20. Dubois Didier, Prade Henry. Possibility theory and its applications: Where do we stand? (Kacprzyk Janusz, Pedrycz Witold, eds.): Springer Berlin Heidelberg; 2015.

21. Ernst O.G., Powell C.E., Silvester D.J., Ullmann E. Efficient solvers for a linear stochastic galerkin mixed formulation of diffusion problems with random data. SIAM Journal on Scientific Computing. 2009;31(2):1424-1447.

22. Falsone G., Impollonia N. A new approach for the stochastic analysis of finite element modelled structures with uncertain parameters. Computer Methods in Applied Mechanics and Engineering. 2002;191(44):5067 -5085. 
23. Galipeau Evan, Castaneda Pedro Ponte. The effect of particle shape and distribution on the macroscopic behavior of magnetoelastic composites. International Journal of Solids and Structures. 2012;49(1):1 -17.

24. Galipeau Evan, Rudykh Stephan, deBotton Gal, Castaneda Pedro Ponte. Magnetoactive elastomers with periodic and random microstructures. International Journal of Solids and Structures. 2014;51(18):3012 -3024.

25. Ghanem R. G., Spanos P. D. Stochastic finite elements: a spectral approach: Dover Publications, inc, New York; 2003.

26. Hadigol Mohammad, Doostan Alireza, Matthies Hermann G., Niekamp Rainer. Partitioned treatment of uncertainty in coupled domain problems: A separated representation approach. Computer Methods in Applied Mechanics and Engineering. 2014;274(0):103 -124.

27. Hanss M. (). A nearly strict fuzzy arithmetic for solving problems with uncertainties, Peachfuzz 2000. 19th international conference of the north american fuzzy information processing society - nafips (cat. no.00th8500), pp. 439-443.

28. Hanss M., Turrin S. A fuzzy-based approach to comprehensive modeling and analysis of systems with epistemic uncertainties. Structural Safety. 2010;32(6):433 -441 Modeling and Analysis of Rare and Imprecise Information.

29. Hanss Michael. The transformation method for the simulation and analysis of systems with uncertain parameters. Fuzzy Sets and Systems. 2002;130(3):277 -289 .

30. Hanss Michael. Applied fuzzy arithmetic: Springer Berlin Heidelberg; 2005.

31. Hanss Michael, Willner Kai. (). On using fuzzy arithmetic to solve problems with uncertain model parameters, Proc. of the euromech, pp. 85-92.

32. Hanss Michael, Willner Kai. A fuzzy arithmetical approach to the solution of finite element problems with uncertain parameters. Mechanics Research Communications. 2000;27(3):257-272.

33. Hiriyur Badri, Waisman Haim, Deodatis George. Uncertainty quantification in homogenization of heterogeneous microstructures modeled by xfem. International Journal for Numerical Methods in Engineering. 2011;88(3):257-278.

34. Javili A., Chatzigeorgiou G., Steinmann P. Computational homogenization in magneto-mechanics. International Journal of Solids and Structures. 2013;50(25-26):4197-4216.

35. Kaestner M., Haasemann G., Ulbricht V. Multiscale xfem-modelling and simulation of the inelastic material behaviour of textile-reinforced polymers. International Journal for Numerical Methods in Engineering. 2011;86(4-5):477-498.

36. Kaestner M., Mueller S., Goldmann J., Spieler C., Brummund J., Ulbricht V. Higher-order extended fem for weak discontinuities — level set representation, quadrature and application to magneto-mechanical problems. International Journal for Numerical Methods in Engineering. 2013;93(13):1403-1424.

37. Kaminski Marcin. The stochastic perturbation method for computational mechanics: A John Wiley \& Sons, Ltd.; 2013.

38. Kaminski Marcin. Homogenization with uncertainty in poisson ratio for polymers with rubber particles. Composites Part B: Engineering. 2015;69(Supplement C):267 -277.

39. Kaminski Marcin, Sokolowski Damian. Dual probabilistic homogenization of the rubber-based composite with random carbon black particle reinforcement. Composite Structures. 2016;140(Supplement C):783 -797.

40. Karavelas Menelaos I., Yvinec Mariette. (). Dynamic additively weighted voronoi diagrams in 2d, Algorithms — esa 2002: 10th annual european symposium rome, italy, september 17-21, 2002 proceedings, pp. 586-598.

41. Klimke A. (). An efficient implementation of the transformation method of fuzzy arithmetic, 22nd international conference of the north american fuzzy information processing society, nafips 2003, pp. 468-473. 
42. Klimke Andreas, Willner Kai, Wohlmuth Barbara. Uncertainty modeling using fuzzy arithmetic based on sparse grids: Applications to dynamic systems. International Journal of Uncertainty, Fuzziness and Knowledge-Based Systems. 2004;12(06):745-759.

43. Kucerova Anna, Sykora Jan, Rosic Bojana, Matthies Hermann G. Acceleration of uncertainty updating in the description of transport processes in heterogeneous materials. Journal of Computational and Applied Mathematics. 2012;236(18):4862 -4872 FEMTEC 2011: 3rd International Conference on Computational Methods in Engineering and Science, May 9-13, 2011.

44. Lang Christapher, Doostan Alireza, Maute Kurt. Extended stochastic fem for diffusion problems with uncertain material interfaces. Computational Mechanics. 2012;51(6):1031-1049.

45. Lang Christapher, Sharma Ashesh, Doostan Alireza, Maute Kurt. Heaviside enriched extended stochastic fem for problems with uncertain material interfaces. Computational Mechanics. 2015;56(5):753-767.

46. Leclerc W., Karamian-Surville P., Vivet A. An efficient stochastic and double-scale model to evaluate the effective elastic properties of $2 \mathrm{~d}$ overlapping random fibre composites. Computational Materials Science. 2013;69(0):481 -493.

47. Legrain G., Cartraud P., Perreard I., Moes N. An x-fem and level set computational approach for image-based modelling: Application to homogenization. International Journal for Numerical Methods in Engineering. 2011;86(7):915-934.

48. Liu G.R., Zeng W., Nguyen-Xuan H. Generalized stochastic cell-based smoothed finite element method (GS-CS-FEM) for solid mechanics. Finite Elements in Analysis and Design. 2013;63(Supplement C):51 -61.

49. Lucas V., Golinval J.-C., Paquay S., Nguyen V.-D., Noels L., Wu L. A stochastic computational multiscale approach; application to mems resonators. Computer Methods in Applied Mechanics and Engineering. 2015;294:141 -167.

50. Ma Juan, Sahraee Shahab, Wriggers Peter, De Lorenzis Laura. Stochastic multiscale homogenization analysis of heterogeneous materials under finite deformations with full uncertainty in the microstructure. Computational Mechanics. 2015;55(5):819-835.

51. Ma Juan, Zhang Jie, Li Liangjie, Wriggers Peter, Sahraee Shahab. Random homogenization analysis for heterogeneous materials with full randomness and correlation in microstructure based on finite element method and monte-carlo method. Computational Mechanics. 2014;54(6):1395-1414.

52. Ma Xiang, Zabaras Nicholas. An adaptive hierarchical sparse grid collocation algorithm for the solution of stochastic differential equations. Journal of Computational Physics. 2009;228(8):3084 -3113.

53. Moens David, Hanss Michael. Non-probabilistic finite element analysis for parametric uncertainty treatment in applied mechanics: Recent advances. Finite Elements in Analysis and Design. 2011;47(1):4 -16 Uncertainty in Structural Dynamics.

54. Moes N., Cloirec M., Cartraud P., Remacle J.-F. A computational approach to handle complex microstructure geometries. Computer Methods in Applied Mechanics and Engineering. 2003;192(28-30):3163 -3177 Multiscale Computational Mechanics for Materials and Structures.

55. Mohan P. Surya, Nair Prasanth B., Keane Andy J. Multi-element stochastic reduced basis methods. Computer Methods in Applied Mechanics and Engineering. 2008;197(17-18):1495 -1506.

56. Moller Bernd, Beer Michael. Fuzzy randomness: Uncertainty in civil engineering and computational mechanics. 1st ed.: Springer-Verlag Berlin Heidelberg; 2004

57. Nair Prasanth B, Keane Andrew J. Stochastic reduced basis methods. AIAA journal. 2002;40(8):1653-1664.

58. Nouy A., Clement A. extended stochastic finite element method for the numerical simulation of heterogeneous materials with random material interfaces. International Journal for Numerical Methods in Engineering. 2010;83(10):1312-1344.

59. Nouy A., Clement A., Schoefs F., Moes N. An extended stochastic finite element method for solving stochastic partial differential equations on random domains. Computer Methods in Applied Mechanics and Engineering. 2008;197(51-52):4663 -4682. 
60. Nouy Anthony. A generalized spectral decomposition technique to solve a class of linear stochastic partial differential equations. Computer Methods in Applied Mechanics and Engineering. 2007;196(45-48):4521 -4537.

61. Nouy Anthony. Generalized spectral decomposition method for solving stochastic finite element equations: Invariant subspace problem and dedicated algorithms. Computer Methods in Applied Mechanics and Engineering. 2008;197(51-52):4718 -4736.

62. Nouy Anthony, Maitre Olivier P. Le. Generalized spectral decomposition for stochastic nonlinear problems. Journal of Computational Physics. 2009;228(1):202 -235.

63. Papoulis Athanasios, Pillai S. Unnikrishna. Probability, random variables and stochastic processes: McGraw-Hill Education; 2001.

64. Pivovarov Dmytro, Steinmann Paul. Modified sfem for computational homogenization of heterogeneous materials with microstructural geometric uncertainties. Computational Mechanics. 2016;57(1):123-147.

65. Pivovarov Dmytro, Steinmann Paul. On stochastic fem based computational homogenization of magneto-active heterogeneous materials with random microstructure. Computational Mechanics. 2016;58(6):981-1002.

66. Rosic B., Matthies H.G. Computational approaches to inelastic media with uncertain parameters. Journal of the Serbian Society for Computational Mechanics. 2008;2(1):28-43.

67. Rosic Bojana, Matthies Hermann, Zivkovic Miroslav. Uncertainty quantification of inifinitesimal elastoplasticity. Scientific Technical Review. 2011;61(2):3-9.

68. Sachdeva Sachin K., Nair Prasanth B., Keane Andy J. Comparative study of projection schemes for stochastic finite element analysis. Computer Methods in Applied Mechanics and Engineering. 2006;195(19-22):2371 -2392.

69. Saeb S., Steinmann P., Javili A. Bounds on size-dependent behaviour of composites. Philosophical Magazine. 2018;98(6):437-463.

70. Saeb Saba, Steinmann Paul, Javili Ali. Aspects of computational homogenization at finite deformations: A unifying review from reuss' to voigt's bound. ASME. Applied Mechanics Reviews. 2016September;68(5):050801-050801-33.

71. Sakata S., Ashida F., Kojima T. Stochastic homogenization analysis on elastic properties of fiber reinforced composites using the equivalent inclusion method and perturbation method. International Journal of Solids and Structures. 2008;45(25-26):6553 -6565.

72. Sarkar Abhijit, Benabbou Nabil, Ghanem Roger. Domain decomposition of stochastic pdes: Theoretical formulations. International Journal for Numerical Methods in Engineering. 2009;77(5):689-701.

73. Savvas Dimitrios, Stefanou George, Papadrakakis Manolis. Determination of rve size for random composites with local volume fraction variation. Computer Methods in Applied Mechanics and Engineering. 2016;305:340 -358.

74. Savvas Dimitris, Stefanou George, Papadrakakis Manolis, Deodatis George. Homogenization of random heterogeneous media with inclusions of arbitrary shape modeled by xfem. Computational Mechanics. 2014;54(5):1221-1235.

75. Scheunemann Lisa, Schroeder Joerg, Balzani Daniel, Brands Dominik. Construction of statistically similar representative volume elements — comparative study regarding different statistical descriptors. Procedia Engineering. 2014;81:1360 -1365 11th International Conference on Technology of Plasticity, ICTP 2014, 19-24 October 2014, Nagoya Congress Center, Nagoya, Japan.

76. Segalman Daniel J., Brake Matthew R., Bergman Lawrence A., Vakakis Alexander F., Willner Kai. (). Epistemic and aleatoric uncertainty in modeling, Asme 2013 international design engineering technical conferences and computers and information in engineering conference, volume 8: 22nd reliability, stress analysis, and failure prevention conference; 25 th conference on mechanical vibration and noise.

77. Shynk John J. Probability, random variables, and random processes: Theory and signal processing applications: Wiley-Interscience; 2012. 
78. Spieler C., Kaestner M., Goldmann J., Brummund J., Ulbricht V. Xfem modeling and homogenization of magnetoactive composites. Acta Mechanica. 2013;224(11):2453-2469 (English).

79. Stefanou G., Nouy A., Clement A. Identification of random shapes from images through polynomial chaos expansion of random level set functions. International Journal for Numerical Methods in Engineering. 2009;79(2):127-155.

80. Stefanou G., Papadrakakis M. Stochastic finite element analysis of shells with combined random material and geometric properties. Computer Methods in Applied Mechanics and Engineering. 2004;193:139-160.

81. Stefanou George. The stochastic finite element method: Past, present and future. Computer Methods in Applied Mechanics and Engineering. 2009;198:1031 -1051 .

82. Stefanou George. Simulation of heterogeneous two-phase media using random fields and level sets. Frontiers of Structural and Civil Engineering. 2014;9(2):114-120.

83. Ullmann Elisabeth, Elman Howard C., Ernst Oliver G. Efficient iterative solvers for stochastic galerkin discretizations of log-transformed random diffusion problems. SIAM J. Sci. Comput. 2012;34(2):659-682.

84. Walz Nico-Philipp. Fuzzy arithmetical methods for possibilistic uncertainty analysis, Schriften aus dem Institut für Technische und Numerische Mechanik der Universität Stuttgart: Shaker Verlag GmbH; 2016.

85. Wan Xiaoliang, Karniadakis George Em. An adaptive multi-element generalized polynomial chaos method for stochastic differential equations. Journal of Computational Physics. 2005;209(2):617 -642.

86. Xu X. Frank. A multiscale stochastic finite element method on elliptic problems involving uncertainties. Computer Methods in Applied Mechanics and Engineering. 2007;196:2723 -2736.

87. Zabihyan R., Mergheim J., Javili A., Steinmann P. Aspects of computational homogenization in magneto-mechanics: Boundary conditions, rve size and microstructure composition. International Journal of Solids and Structures. 2018;130-131:105 -121.

88. Zaccardi C., Chamoin L., Cottereau R., Ben Dhia H. Error estimation and model adaptation for a stochastic-deterministic coupling method based on the arlequin framework. International Journal for Numerical Methods in Engineering. 2013;96(2):87-109.

89. Zadeh L.A. Fuzzy sets as a basis for a theory of possibility. Fuzzy Sets and Systems. 1978;1(1):3 -28.

90. Zadeh Lotfi A. Fuzzy sets. Information and control. 1965;8(3):338-353. 
<smiles>C1CCC1</smiles> 